\title{
MicroRNA as a Therapeutic Target in Cardiac Remodeling
}

\author{
Chao Chen, Murugavel Ponnusamy, Cuiyun Liu, Jinning Gao, Kun Wang, and Peifeng Li \\ Center for Developmental Cardiology, Institute for Translational Medicine, Qingdao University, Dengzhou Road 38, \\ Qingdao 266021, China \\ Correspondence should be addressed to Kun Wang; wangk696@163.com and Peifeng Li; peifli@qdu.edu.cn
}

Received 3 May 2017; Revised 23 July 2017; Accepted 9 August 2017; Published 28 September 2017

Academic Editor: Kai Chen

Copyright (C) 2017 Chao Chen et al. This is an open access article distributed under the Creative Commons Attribution License, which permits unrestricted use, distribution, and reproduction in any medium, provided the original work is properly cited.

MicroRNAs (miRNAs) are small RNA molecules that contain 18-25 nucleotides. The alterations in their expression level play crucial role in the development of many disorders including heart diseases. Myocardial remodeling is the final pathological consequence of a variety of myocardial diseases. miRNAs have central role in regulating pathogenesis of myocardial remodeling by modulating cardiac hypertrophy, cardiomyocytes injury, cardiac fibrosis, angiogenesis, and inflammatory response through multiple mechanisms. The balancing and tight regulation of different miRNAs is a key to drive the cellular events towards functional recovery and any fall in this leads to detrimental effect on cardiac function following various insults. In this review, we discuss the impact of alterations of miRNAs expression on cardiac hypertrophy, cardiomyocytes injury, cardiac fibrosis, angiogenesis, and inflammatory response. We have also described the targets (receptors, signaling molecules, transcription factors, etc.) of miRNAs on which they act to promote or attenuate cardiac remodeling processes in different type cells of cardiac tissues.

\section{Introduction}

Myocardial remodeling is one of the major alterations of architecture of the heart that causes the hemodynamic imbalance under pathological stimuli and biomechanical stresses of external stimuli. Myocardial remodeling is a detrimental cardiac problem due to the reason that it is associated with nearly all kinds of heart diseases. The myocardial remodeling process includes physiological changes in structure, dimensions, mass, shape, and functions of the heart as well as cardiac cells. The major contributors in the pathological of myocardial remodeling are cardiac hypertrophy, cardiomyocytes injury, cardiac fibrosis, angiogenesis, and inflammatory response. It is well known that a significant loss of cardiomyocytes due to various insults and slower rate of cardiomyocyte regeneration is the initiative event for the myocardial remodeling in human as well as in animal models [1]. The cardiac inflammation following the myocardial infarction and ischemia reperfusion injury is the primary driving force of delayed cardiac regeneration and pathological cardiac remodeling. The production of extracellular matrix components (ECM) is essential to provide mechanical support to the regenerating heart after cardiac injury. However, the dynamic change in ECM production forces the heart tissue to maladaptive repair, prolonged inflammatory response, and loss of cardiomyocytes, which ultimately leads to cardiac remodeling. The relationship between the risk factors for cardiovascular diseases and myocardial remodeling is shown in Figure 1.

miRNAs are transcripts of noncoding region of the gene. The miRNA era began in 1993 when Lee et al. found the first microRNA in nematode. This field of research has made a tremendous progress in recent years and now it is realized that miRNAs have unavoidable role in both physiological as well as pathological processes [2]. The precursor molecules of miRNAs are called primary miRNAs (pre-miRNAs), which are derived as long fragments in the nucleus by RNA polymerase II. The pre-miRNAs are translocated to the cytoplasm and subsequently processed by different molecular machineries including RNase III-type endonuclease and Dicer, to form a mature double-stranded miRNAs. In maturation process, the pre-miRNAs lose their terminal base pairs and their hairpin structure in order to generate a mature miRNA. The single-stranded form is a fully active mature miRNA, which binds with RNA-induced silencing complexes (RISCs) and specifically binding with its target genes. These binding silences target gene activity at posttranscriptional level $[3,4]$. 


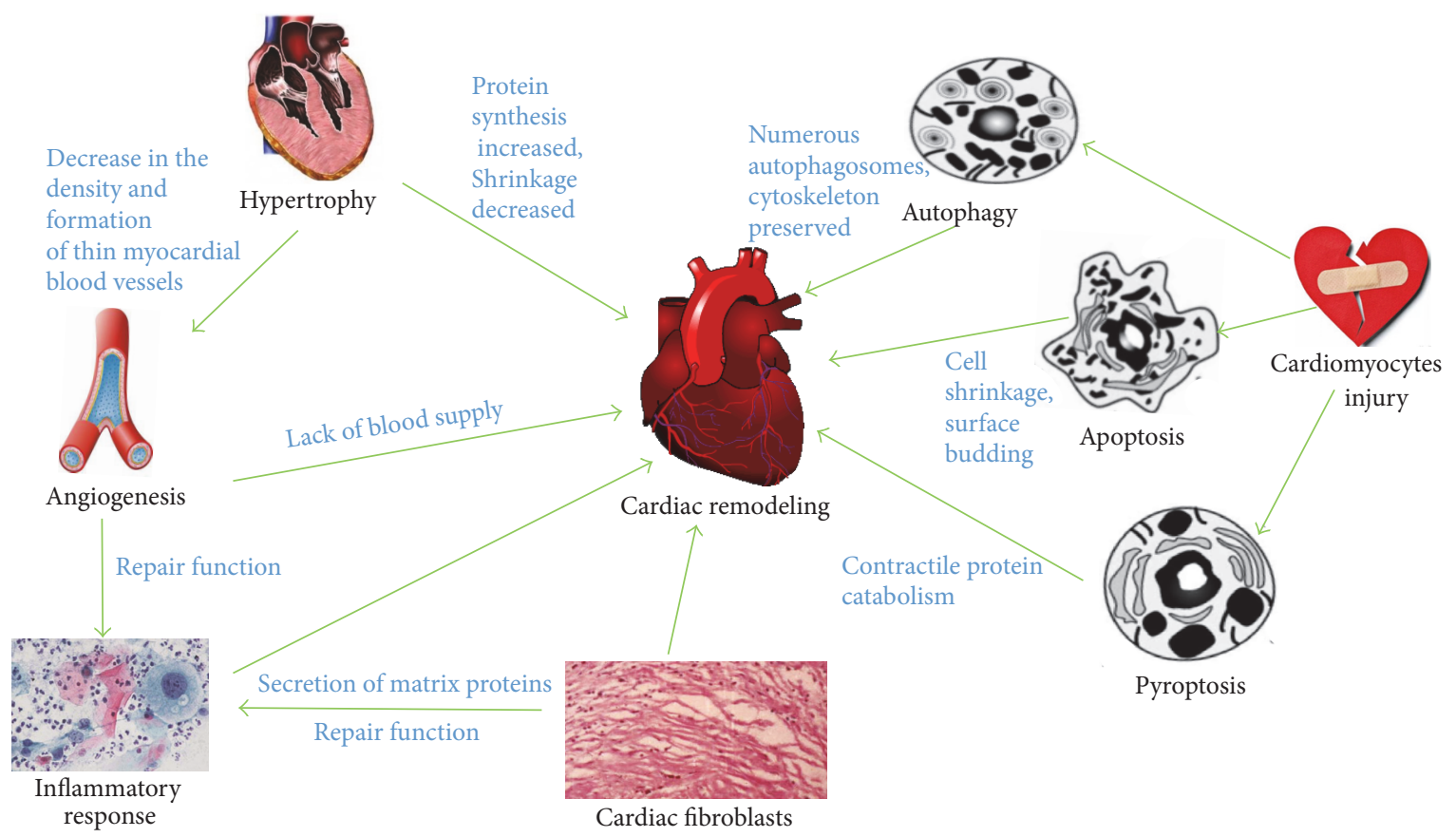

FIgURE 1: The risk factors for cardiovascular diseases and myocardial remodeling.

Many experimental and clinical studies reveal that the expression of miR is highly altered in various cardiovascular problems and their alterations are linked with modulation of activity and expression of key components of cardiomyocyte growth, survival, and death. Thus, miRNAs are considered as a central part of the development of various cardiac disorders, which subsequently leads to cardiac remodeling and progression to heart failure. In this review, we discuss the impact of alterations of miR expression on the process of pathological cardiac remodeling. For the clear illustration, we divide the adverse cardiac remodeling into the following components: cardiac hypertrophy, cardiac fibrosis, myocardial cell injury, and angiogenesis, which are closely related to the progression of cardiac remodeling and heart failure.

\section{2. miRNA and Cardiac Hypertrophy}

Cardiac hypertrophy is one of the major responses to various physiological and pathological stress stimuli in the heart. The major feature of hypertrophic response is an increase in the thickness of the ventricular wall, cardiac dilatation, and heart failure. As the maladaptive hypertrophic response consequently leads to various cardiac problems, many scientific works endeavor to figure out the underlying molecular mechanism of pathological hypertrophy. However, the molecular mechanisms associated with cardiac hypertrophy remain largely unknown $[5,6]$. miRNAs play an important role in regulating the cardiac hypertrophic processes. They either negatively or positively modulate hypertrophic signaling and thus indirectly influence the hypertrophy associated cardiac remodeling.

MiR-1 is one of the key regulators of pathological cardiac hypertrophy by targeting multiple signaling molecules in human as well as in experimental animal models. MiR-1 protects heart structure and functions against cardiac hypertrophic responses by directly targeting and inhibiting translation of many signaling molecules including eukaryotic initiation factor 4E (Eif4e), Mef2a, Gata4, and histone deacetylase 6 (HDAC6) [7-9]. MiR-1 also attenuates calcium signaling dependent cardiac hypertrophic response by negatively regulating calmodulin (CAM), one of the key components of calcium signaling, which contributes to the progression of pathological hypotrophy in the heart [7]. The increased circulatory level of heart specific fat binding protein 2 (FABP3) is an indicator of heart enlargement and hypertrophy in patients and miR-1 can directly target FABP3 in cardiomyocytes. A study found that there is an inverse correlation between the expression of miR-1 in myocardial tissue and FABP3 level in circulation [10]. Another research group found that miR-1 inhibits fibulin-2 (Fbln2) expression and thereby it abolishes activation of TGF $\beta$ signaling and extracellular matrix remodeling in hypertrophic heart $[11,12]$. MiR-133 is one of the abundantly expressed antihypertrophic miR in both animal and human myocardial tissues. MiR133 blocks hyperthyroidism induced cardiac hypertrophy by suppressing the expression of type 1 angiotensin II receptor (AT1R) [13]. Carè et al. (2007) observed that miR-133a attenuates cardiac remodeling by regulating Akt and its downstream signaling molecules such as Cdc42, Rho-A, and Nelf-A/WHSC2 [14]. MiR-10a [15] and miR-497 [16] exert their antihypertrophic effect by blocking translation of Tbx2 and Sirt4, respectively. MiR-223 directly targets cardiac troponin I-interacting kinase (TNNI3K) and inhibits troponin I (cTnI) phosphorylation in cardiomyocytes, which promotes contractility and accumulation of intracellular $\mathrm{Ca}^{2+}$ [17]. MiR-455 is an important miR in the developing heart 
that targets calreticulin to prevent pressure overload induced hypertrophy [18]. MiR-378 suppresses the expression and activities of many growth promoting receptors and signaling pathways, including insulin-like growth factor receptor 1 (IGFR1), growth factor receptor bound protein 2 (Grb2), kinase suppressor of Ras 1 (Ksrl), Ras activity, PI3K-Akt pathway, Mapk1-MAPK signaling, and Raf1-MEK1-ERK1/2 pathway $[19,20]$ This inhibition is associated with reduction of hypertrophic response in cardiac tissue. MiR-145 showed its negative effect in isoproterenol-induced cellular hypertrophy via regulation of the expression of GATA binding protein 6 (GATA6) [21, 22]. MiR-155 alleviates cardiac hypertrophy and improves heart function by repressing the expression of angiotensin II receptor type 1 (AGTR1) and suppression of its downstream calcium signaling pathways [23]. MiR-212/132 family [24] and miR-23a [25] target Foxo3 transcription factor to mitigate the hypertrophic signal in cardiomyocytes. MiR-29a-3p inhibits ET-1-induced hypertrophic response in cardiomyocytes by directly targeting $3^{\prime}$ UTR of NFATc4 [26].

In opposite, many miRNAs play prohypertrophic role in the heart. For instance, miR-208a is a well expressed miR in the heart and it is highly associated with development of cardiac hypertrophy and fibrosis. Hyperthyroidism induces cardiac hypertrophy by promoting the expression of angiotensin type 1 receptor (AT1R). The stimulation of $\alpha$-MHC mediated expression of miR-208a promotes the expression of AT1R in hyperthyroid condition [27]. Another study found that miR208 regulates the translation of thyroid hormone receptor associated protein 1 (THRAP1) and leads to hyperthyroidism under stress condition. This event consequently enhances $\beta$ MHC expression and further aggravates cardiac hypertrophy [28]. MiR-124 effectively represses AngII-induced neonatal cardiomyocytes hypertrophy by blocking the expression of calreticulin (CRT) and glucose-regulated protein (Grp78), an endoplasmic reticulum (ER) stress marker [29]. Several miRNAs reduce the expression of antihypertrophic factors and indirectly contribute to elevation of pathological hypertrophy. MiR-297 negatively regulates the expression of Sigma-1 receptor (Sig-1R) and activates ER stress signaling [30], while miR-17-3p targets metallopeptidase inhibitor 3 (TIMP3), a negative regulator of PTEN-Akt pathway, and resulting in cardiomyocyte hypertrophy [31]. MiR-21 targets suppressors of hypertrophic response by inhibiting translation of $\mathrm{SH} 3$ domain-containing protein 2 (SORBS2), PDZ, and LIM domain 5 (PDLIM5) [32]. MiR-155 acts as a prohypertrophic factor by abolishing the expression of Mef2A and Jarid2 in cardiomyocytes and inhibition of miR-155 improves the cardiac function from hypertrophy induced progressive heart failure [33]. MiR-22 acts as a prohypertrophic factor by targeting PTEN, Sirtuin 1 (Sirtl), and histone deacetylase 4 (Hdac4), which are negative regulators of hypertrophic signaling $[25,34,35]$. Interestingly, miR-23a has mutual regulatory role with lysophosphatidic acid (LPA) receptor. LPA can upregulate miR-23a expression by activation of LPA3 dependent PI3K/Akt pathway [36].

In experimental cardiac hypertrophy, MiR-195 downregulates the translation of HMGAl by targeting its $3^{\prime}$ UTR and this inhibition promotes hypertrophic response [37, 38]. Atrogin 1 and muscle RING finger protein 1 (Murfl) are antihypertrophic factors and their expression is downregulated by miR-19a/b family. miR-19b mediates hypertrophic process by activating the PI3K and calcineurin/nuclear factor of activated T cells (NFAT) signaling, which are prohypertrophic pathway that changes phonotype of cardiomyocytes. Interestingly, miR-19b also has the capability to promote cell survival by attenuating the upregulation of NFAT target gene encoding $\alpha$-crystallin-B and repression of the proapoptotic gene Bim (Bcl-2-interacting mediator of cell death) caused by endoplasmic reticulum (ER) stress [39]. A study in experimental model of hypertrophy found that miR-27b blocks the expression of peroxisome proliferator-activated receptor $\gamma$ (PPAR- $\gamma$ ), which can relieve cardiac hypertrophy mediated cardiac dysfunction and heart failure. In pressure overload model of hypertrophy, it was confirmed that antagonists of miR-27b can restore the expression of PPAR- $\gamma$ and alleviate heart dysfunction in those animals. Surprisingly, transforming growth factor $\beta 1$ (TGF $\beta 1$ ), a hypertrophy stimulant, can regulate the expression of miR-27b. [40]. Sarcoplasmic reticulum Ca2+ ATPase (SERCA2a) is a key calcium transporter responsible for the $\mathrm{Ca}^{2+}$ reuptake, which is suppressed by miR-328 during hypertrophic response in cardiomyocytes as well as in hearts of mouse. This leads to upregulation of the calcineurin/NFAT signaling and cardiac dysfunction [41]. Several experimental studies also found that the suppression of expression of hypertrophy associated miRNAs using antagonists (antagomir) attenuates pathological remodeling in the heart. miR-185 favours hypertrophic response by upregulating multiple genes in calcium signaling pathways, including $\mathrm{Ca}^{2+} / \mathrm{CaM}$ dependent protein kinase II $\delta$ (Camk2d), sodium/calcium exchanger 1 ( $\mathrm{Ncxl}$ ), nuclear factor of activated $\mathrm{T}$ cells, and cytoplasmic calcineurin dependent 3 (Nfatc3) and downregulation of miR-185 can alleviate hypertrophy induced pathological changes in the heart [42]. Similarly, antagonist of miR-101 attenuates experimental cardiac hypertrophy by target the expression of rasrelated protein-1A (Rab1A) [43]. The antimir of miR-208a attenuates cardiac remodeling by upregulation of expression Myh7 in pressure overload induced heart failure in rats [44]. Together, these studies reveal that the balance in the expression of certain miRNAs is essential to control the hypertrophic response in cardiomyocytes and preservation of cardiac structure and function. The known microRNAs (miRNAs) in cardiac hypertrophic and their target genes are summarized in Figure 2 and Table 1.

\section{3. miRNA and Fibrosis}

Cardiac fibroblast is the most abundant cell type in the heart that comprises about two-thirds of the total number of cardiac cell types. The transient activation and proliferation of cardiac fibroblasts after cardiac injury are vital to maintain cardiac integrity and function. However, the persistence of fibroblast activation process accumulates myofibroblast phenotype in the cardiac tissue and that causes adverse myocardial remodeling and cardiac dysfunction. The relentless availability of growth factors, abundant expression of growth factor receptors, and accumulation of cytoplasmic 


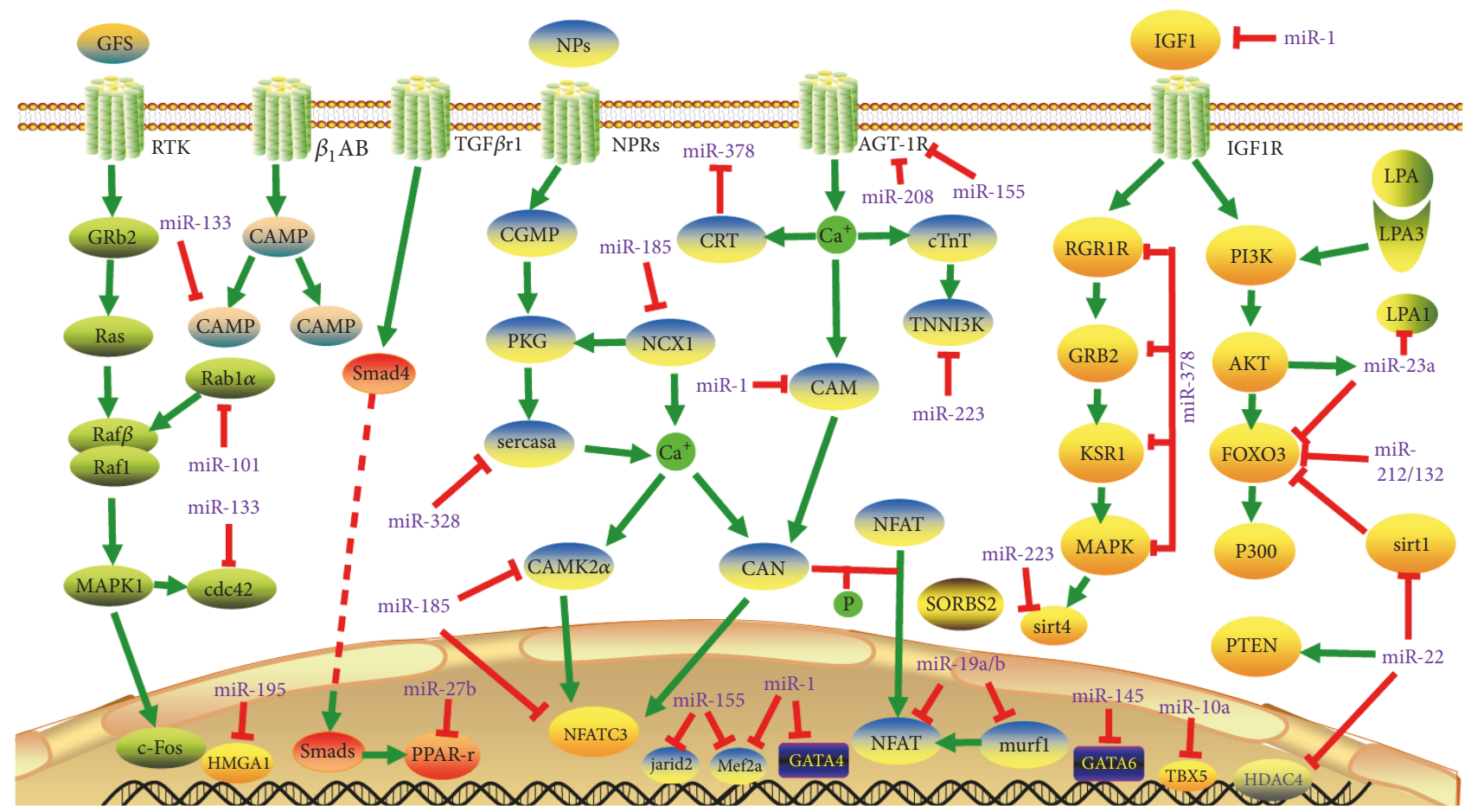

FIGURE 2: MicroRNAs (miRNAs) in cardiac hypertrophic pathways. Arrows colored in red indicate the functions of depression; arrows colored in green indicate the functions of activation. Abbreviations for mRNAs: AGTR1: angiotensin II receptor type 1; Camk2d: calcium/calmodulindependent protein kinase II delta; Cdc42: cell division cycle 42; c-Fos: proto-oncogene protein; CRT: calreticulin; Foxo3: Forkhead box O3; Gata4: GATA binding protein 4; GATA6: GATA binding protein 6; Grb2: growth factor receptor bound protein 2; Hdac4: histone deacetylase 4; HMGA1: high mobility group AT-hook 1; NFAT: nuclear factor of activated T cells; IGF-1: insulin-like growth factor 1; IGF1R: insulin-like growth factor 1 receptor; Jarid2: jumonji and AT-rich interaction domain-containing protein 2; Ksr1: kinase suppressor of ras 1; LPA1: lysophosphatidic acid receptor 1; Mapk1: mitogen-activated protein kinase 1; Murf1: tripartite motif-containing 63; NCX1: sodium/calcium exchanger 1; Nfatc3: nuclear factor of activated T cells, cytoplasmic, calcineurin dependent 3; Ppar $\gamma$ : peroxisome proliferatoractivated receptor $\gamma$; PTEN: phosphatase and tensin homolog; p300: E1A binding protein p300; Rab1A: Ras-related protein Rab la; Serca2a: Sarco/endoplasmic reticulum $\mathrm{Ca}^{2+}$-ATPase 2a; SIRT1: Sirtuin 1; SMAD4: SMAD family member 4; SORBS2: SH3 domain-containing protein 2; TGF- $\beta$ R1: transforming growth factor $\beta$ receptor 1; TNNI3K: troponin I type 3 interacting kinase; cAMP: cyclic adenosine monophosphate; cGMP: cyclic guanosine monophosphate; PKG: cGMP-dependent protein kinase; CAM: calmodulin; CTnT: cardiac troponin T; NPs: natriuretic peptides.

matrix proteins play a fundamental role in the activation of cardiac fibroblast and fibrotic processes $[45,46]$. The other factors such as reactive oxygen species (ROS), inflammatory cytokines and chemokines, and mast cell-derived proteases also play crucial role in the development of myocardial fibrosis (Figure 2). Many miRNAs have influence on fibrogenesis either positively or negatively by modulating the expression/activities of fibrotic signaling molecules in the cardiac tissue.

Transforming growth factor $\beta$ (TGF $\beta$ ) is a multifunctional cytokine that plays a central role in the development and progression of fibrosis in many tissues including heart. The activation TGF $\beta$ dependent signaling pathway and relentless expression of TGF $\beta$ signaling components play crucial role in the expression of extracellular matrix components (ECM) and aggravation of fibrotic response, which consequently leads to ventricular remodeling in the heart. Several miRNAs exert their antifibrotic activity by directly antagonizing the expression and/or activity of TGF $\beta$ signaling components in cardiac myofibroblasts. MiR-101 blocks excessive production of matrix proteins and proliferation of activated fibroblasts by suppressing expression of TGF $\beta$ RI and c-Fos. In experimental model, overexpression of miR-101 alleviates deterioration of cardiac performance caused by fibrotic process $[47,48]$. MiR-122 can control the expression of TGF- $\beta 1$, but its expression is downregulated in patients and leads to severe myocardial fibrosis [49]. MiR24 inhibits TGF $\beta$-smad $2 / 3$ signaling mediated differentiation and migration of cardiac fibroblasts by inhibiting furin, a regulatory protein involved in latent $\mathrm{TGF} \beta$ activation process. A synthetic precursor of miR-24 can improve heart function [50]. Similarly, MiR-98 and miR-19a-3p/19b-3p inhibit fibrogenesis by blocking the expression of TGF $\beta$ RI and TGF $\beta$ RII, respectively. These receptors are integral part of ECM production, cardiac fibrosis, and ventricular thickening [51, 52]. The miR-15 family is one of the most important miRNAs involved in the regulation of fibrosis. It encodes six miRNAs (miR-15a, miR-15b, miR-16, miR-195, miR-497, and miR-322). The expression of multiple miR-15 family members targets TGF- $\beta$ R1, p38, SMAD3, SMAD7, and 
TABLE 1: Summary of reported miRNAs and their targets in cardiac hypertrophy.

\begin{tabular}{|c|c|c|c|c|}
\hline miRNAs & Targets & Effector cells & Signal pathways & References \\
\hline \multirow{9}{*}{ miR-1 } & & Antihypertrophic & & \multirow{9}{*}[7-12]{} \\
\hline & Eif4e & $\mathrm{C} 57 \mathrm{Bl} / 6$ mouse and NMCMs & Translation & \\
\hline & Cdk6 & NRVCs and ACC mouse & CDKs-Rb pathway & \\
\hline & HDAC6 & NRCMs and Wistar rats & Chromatin modifying & \\
\hline & CAM & TG mouse and NRVMs & Calcium signaling & \\
\hline & Mef2a & TG mouse and NRVMs & Calcium signaling & \\
\hline & Gata4 & TG mouse and NRVMs & Calcium signaling & \\
\hline & FABP3 & NMCMs and TAC mouse & PPAR & \\
\hline & Fbln 2 & NMCMs & ECM & \\
\hline miR-10a & Tbx5 & TAC mouse and NRVMs & transcription & {$[15]$} \\
\hline miR-497 & Sirt4 & TAC mouse and NRVMs & AMPK & {$[16]$} \\
\hline \multirow{4}{*}{ miR-133 } & AT1R & Male Wistar rats and NRCMs & ERK-MAPK & \multirow{4}{*}[13,14]{} \\
\hline & Cdc42, & NMCMs and TAC mouse & MAPK & \\
\hline & Rho-A, & NMCMs and TAC mouse & cGMP-PKG & \\
\hline & Nelf-A/WHSC2 & NMCMs and TAC mouse & Transcription & \\
\hline miR-223 & TNNI3K & NRCMs and TAC mouse & Calcium signaling & {$[17]$} \\
\hline miR-455 & CRT & TAC mouse & Calcium signaling & {$[18]$} \\
\hline \multirow{4}{*}{ miR-378 } & Mapk1 & NRCMs and TAC mouse & MAPK & \multirow{4}{*}[19,20]{} \\
\hline & IGF1r & NRCMs and TAC mouse & MAPK & \\
\hline & Grb2 & NRCMs and TAC mouse & MAPK & \\
\hline & Ksrl & NRCMs and TAC mouse & MAPK & \\
\hline miR-145 & GATA6 & NRCMs and TAC mouse & cGMP-PKG & {$[21,22]$} \\
\hline miR-29a-3p & NFATc4 & H9c2 cell & cGMP-PKG & {$[26]$} \\
\hline \multirow{4}{*}{$\operatorname{miR}-21-3 p$} & & Prohypertrophic & & \multirow{4}{*}[32]{} \\
\hline & SORBS2 & NRVMs and NRCFs & Transcription & \\
\hline & PDZ & NRVMs and NRCFs & Transcription & \\
\hline & PDLIM5 & NRVMs and NRCFs & Transcription & \\
\hline \multirow{3}{*}{ miR-208 } & AT1R & Male wistar rats and NRCMs & ERK-MAPK & \multirow{3}{*}[27,28,44]{} \\
\hline & THRAP1 & TG mouse & Thyroid hormone & \\
\hline & Myh7 & Adult male Dahl salt-sensitive rats & & \\
\hline \multirow[t]{2}{*}{ miR-124 } & Grp78 & NRCMs & $\begin{array}{l}\text { Reticulum (ER) stress } \\
\text { signaling }\end{array}$ & \multirow{2}{*}[29]{} \\
\hline & CRT & NRCMs & Calcium signaling & \\
\hline miR-297 & Sig-1R & TAC rat and NRVMs & ER stress signaling & [30] \\
\hline miR-17-3p & TIMP3 & NRVMs and C57BL/6 mice & PTEN-AKT pathway & [31] \\
\hline \multirow{3}{*}{ miR-155 } & Mef2A & TAC rat and KO mice & Calcineurin pathway & \multirow{3}{*}[23,33]{} \\
\hline & Jarid2 & TAC rat and $\mathrm{KO}$ mice & Calcineurin pathway & \\
\hline & AGTR1 & Rat H9C2 cell & $\begin{array}{c}\text { Calcium signaling } \\
\text { pathways }\end{array}$ & \\
\hline miR-212/132 & Foxo3 & H9c2 cells and TAC mouse & PI3K-Akt & {$[24]$} \\
\hline \multirow{3}{*}{ miR-22 } & Hdac4 & $\begin{array}{c}\text { NRVCs, miR-22 } \\
\text { KO mouse }\end{array}$ & AMPK & \multirow{3}{*}[25,34,35]{} \\
\hline & Pten & NRVCs & PI3K-AKT & \\
\hline & Sirtl & $\begin{array}{l}\text { NRVCs, miR-22 } \\
\text { KO mouse }\end{array}$ & AMPK & \\
\hline \multirow{2}{*}{ miR-23a } & Foxo3a, & NMCMs, TAC and TG mouse & PI3K-AKT & \multirow{2}{*}[25,36]{} \\
\hline & LPA1 & NMCMs & PI3K-AKT & \\
\hline miR-195 & HMGA1 & NRVCs & & {$[37,38]$} \\
\hline \multirow{2}{*}{$\mathrm{miR}-19 \mathrm{a} / \mathrm{b}$} & atroginl, & NRVCs and TAC mouse & calcineurin/NFAT & \multirow[b]{2}{*}[39]{} \\
\hline & Murf1 & NRVCs and TAC mouse & PKC & \\
\hline miR-27b & Ppar $\gamma$ & NRVCs, TAC and TG mouse & PPAR & {$[40]$} \\
\hline
\end{tabular}


TABle 1: Continued.

\begin{tabular}{ccccc}
\hline miRNAs & Targets & Effector cells & Signal pathways & References \\
\hline miR-328 & Serca2a & NRVCs, TAC and TG mouse & cGMP-PKG & Calcium \\
\hline miR-185 & Camk2d & NRVMs and TAC mouse & cGMP-PKG & [42] \\
\hline \multirow{2}{*}{ miR-101 } & Ncx1 & NRVMs and TAC mouse & cGMP-PKG & MAPKK \\
& Nfatc3 & NRVMs and TAC mouse & TAC rat & M3] \\
\hline
\end{tabular}

LPA1: lysophosphatidic acid receptor 1; Mapk1: mitogen-activated protein kinase 1; Murf1: tripartite motif-containing 63; NCX1: sodium/calcium exchanger 1; Nfatc3: nuclear factor of activated T cells, cytoplasmic, calcineurin dependent 3; NFATc4: nuclear factor of activated T cells 4; PDLIM5: PDZ and LIM domain 5; Ppar $\gamma$ : peroxisome proliferator-activated receptor $\gamma$; p300: E1A binding protein p300; Rab1A: Ras-related protein Rab la; RasGAP: Ras GTPase-activating protein; Rheb: Ras homolog enriched in brain; Rho-A: Ras homolog family member A; Serca2a: Sarco/endoplasmic reticulum Ca ${ }^{2+}$-ATPase 2a; SORBS2: SH3 domain-containing protein 2; Sox6: sex-determining region Y box 6; THRAP1: mediator complex subunit 13; TNNI3K: troponin I type 3 interacting kinase; NRVCs: neonatal rat ventricular cardiomyocytes; AAC: abdominal aortic constriction; TBX5: T-box 5; NRCMs: neonatal rat cardiomyocytes; SORBS2: SH3 domain-containing protein 2; PDLIM5: PDZ and LIM domain 5; NRVMs: neonatal rat ventricular myocytes; NRCFs: neonatal rat cardiac fibroblasts.

endoglin to inhibits the TGF- $\beta$-pathway. However, the family of miR-15 has negative effect on cardiomyocyte proliferation $[53,54]$. Likewise, miR-378 attenuates TGF/Smad/Ras signaling by suppressing the expression of several profibrotic transcription factors such as AP1 transcription factors, cFos, and c-Jun in cardiac fibroblasts. However, this miRNA expression is downregulated in cardiac tissue during fibrotic process by an unknown mechanism [55]. Several miRNAs play profibrotic role by upregulating the expression of TGF $\beta$ signaling molecules. miR-21 is highly expressed by cardiac fibroblasts in postmyocardial injury condition and its expression accelerates fibrotic process by blocking the expression of TGF $\beta$ RIII, which is a negative regulator of TGF $\beta 1-\mathrm{Smad} 3$ signaling [56]. miR-21 also favours fibrogenic process by inhibiting the expression of sprouty homolog 1 (Spryl), a molecule required for controlling fibroblast growth factor secretion and inhibition of ERK-MAP kinase activity. miR155 increases TGF $\beta 1-S m a d 2$ signaling pathway by elevating the level of Smad 2 and resulting in cardiac fibrosis [57]. TGF $\beta 1$ induces expression of miR-22, which increases the level of Smad4, which is an essential component of Smad complex $(\operatorname{Smad} 2 / 3 / 4)$ that facilitates the translocation to nucleus and induction of downstream genes [58].

Connective tissue growth factor (CTGF) is a key molecule involved in the development and progression of fibrosis by promoting the synthesis of ECM proteins including collagen. miR-133 and miR-30 downregulate CTGF expression and attenuate cardiac fibrosis [59]. miR-26a also attenuates fibrotic process by directly targeting translation of CTGF genes and expression of collagen type I. Interestingly, NF-kB and miR-26a reciprocally regulate each other in the fibrotic heart. The increased expression of NF-kB diminishes levels of miR-26a, while overexpression of miR-26a diminishes NF-kB activation [60]. A recent study found that miR29 acts as an antifibrotic factor by accelerating the expression of multiple extracellular matrix genes including elastin [61], fibrillin 1 (Fbn1), collagen type I, $\alpha 1$ and 2 (Coll $\alpha 1$, Coll $\alpha 2)$, collagen type III, $\alpha 1$ (Col3 $\alpha 1)$, and fibronectin (FBN) [62, 63]. Another research group also confirmed the antifibrotic effects of miR$29 \mathrm{~b}$ in angiotensin II induced cardiac fibrosis model [64]. MiR-146a targets VEGF expression and suppresses VEGF dependent proliferation and expansion myofibroblasts [65]. The other miRNAs such as miR-142-3p and miR-433 target high mobility group box 1 (HMGB1), AZIN1, and JNK1, respectively, to ameliorate cardiac fibrosis [66, 67].

Several miRNAs positively modulate myocardial fibrosis by directly upregulating the expression of profibrotic genes or indirectly through suppressing the expression of antifibrotic signaling molecules, which inhibit fibrotic process by either negatively regulating profibrotic factors activity or expression. miR-208 is a cardiac-specific miR whose upregulation is closely related to the development of cardiac fibrosis. miR208 induces myocardial fibrosis by upregulating the expression of endoglin, a coreceptor of TGF and $\beta$-myosin heavy chain ( $\beta$-MHC) [68, 69]. A molecular study found that miR208 specifically contributes to stress dependent expression of $\beta$-MHC and cardiac fibrosis. This is partly due to suppression of thyroid hormone receptor associated protein 1 (THRAP1), a coregulator of thyroid receptor, which can positively modulate $\alpha$-MHC gene expression and suppression of $\beta$-MHC in cardiac tissue [28]. Interestingly, miR-22 acts as a downstream of TGF $\beta 1$ signaling. TGF $\beta 1$ induces expression of miR-22, which increases the level of Smad4, an essential component of Smad complex (Smad2/3/4) that facilitates the translocation to nucleus and induction of downstream genes [58]. miR-499 plays a crucial role in the development of myocardial hypertrophy and fibrosis by targeting many intracellular signaling molecules and transcription factors including Akt, MAPKs, Egrl, Egr2, and Fos or promoting Myh7 and Actal expressions [68]. miR-125b acts as a profibrotic molecule by targeting apelin, which is one of the key suppressors of cardiovascular remodeling that inhibits TGF $\beta$ mediated collagen production and Ang II induced plasminogen activator inhibitor type I (PAI-1) [70, 71]. Collectively, these studies reveal that miRNAs play unavoidable role in both controlling and activating fibrogenic processes and cardiac remodeling. The balancing of antifibrotic miRNAs and profibrotic miRNAs is crucial to determine the fibroblast activation, acceleration of fibrotic signaling, accumulation of ECM components, and fibrotic processes. The known miRNAs in cardiac fibrosis and their target genes are summarized in Figure 3 and Table 2. 


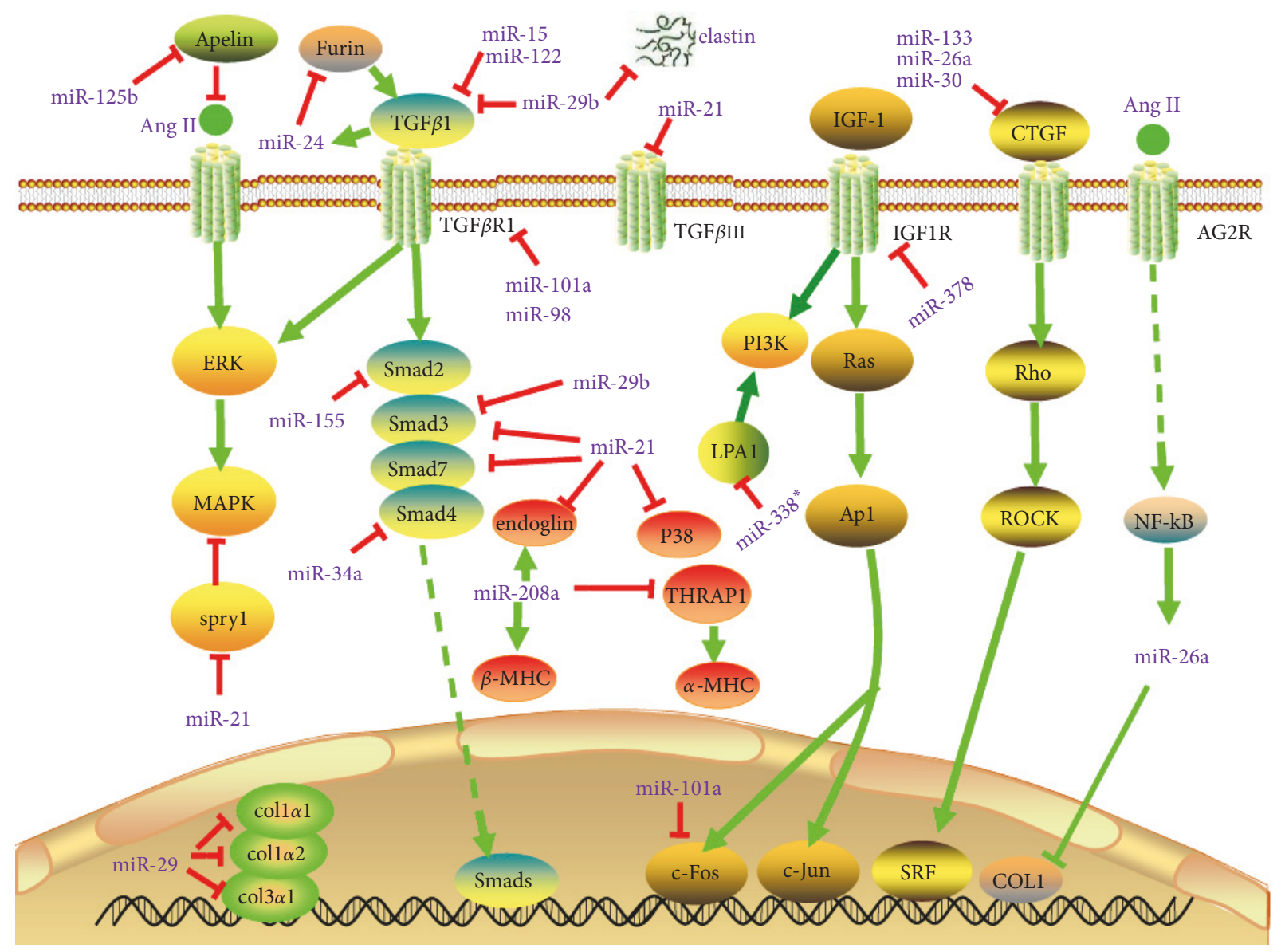

Figure 3: MicroRNAs (miRNAs) in cardiac fibrosis pathways. Arrows colored in red indicate the functions of depression; arrows colored in green indicate the functions of activation. Abbreviations for mRNAs: $\beta$-MHC: beta myosin heavy chain; COL1: collagen, type 1; Coll $\alpha$ : collagen, type $1 \alpha 1$; Coll $\alpha 2$ : collagen, type $1 \alpha 2$; Col3 $\alpha 1$ : collagen, type $3 \alpha 1$; CTGF: connective tissue growth factor; IGF-1: insulin-like growth factor 1; IGF-1R: insulin-like growth factor 1 receptor; $\mathrm{p} 38$ : Tumor protein p38; ROCK1: Rho associated coiled-coil containing protein kinase 1; SMAD3: SMAD family member 3; SMAD2: SMAD family member 2; SMAD7: SMAD family member 7; Spryl: sprouty homolog 1; TGF$\beta 1$ : transforming growth factor $\beta$ 1; TGF- $\beta$ R1: transforming growth factor $\beta$ receptor 1 ; TGF $\beta$ RIII: transforming growth factor $\beta$ receptor III; THRAP1: mediator complex subunit 13; ERK: extracellular regulated protein kinases; MAPK: mitogen-activated protein kinase; AG2R: anterior gradient-2.

\section{4. miRNA and Cardiomyocytes Injury}

The loss of cardiomyocytes due to injury and their low regenerative capability in the adult heart is the early event responsible for the ventricular dysfunction, fibrosis, and progressive heart failure. Many studies found that chronic cardiac remodeling is highly associated with increasing cardiomyocyte cell death. The damage and/or loss of cardiomyocytes occur in various cardiovascular problems including myocardial infarction, ischemia/reperfusion injury, cardiomyopathy, and cardiac toxicity. However, the mode of cell death mainly depends on the type of insult to the heart. In the last few years, numerous miRNAs have been identified as regulators of cardiomyocytes injury due to their role in apoptosis, autophagy, and inflammatory response. In this part, we summarize the influence of miRNAs on different mode of cell death in cardiomyocytes.
4.1. miRNA and Apoptosis. Apoptosis is the major form of cardiomyocyte death in the failing heart. In cardiomyocytes, apoptosis can be activated by both intrinsic pathway (mediated by mitochondria) and extrinsic pathway (mediated by plasma membrane death receptors). However, these two pathways converge into mitochondria to induce the release of mitochondrial apoptogenic molecules and activation of caspase cascades. The activation of apoptosis is a complex event, which is tuned by highly regulated interaction of proapoptotic molecules. Many cell signaling pathways including the phosphatidylinositol 3-kinase (PI3K)/Akt pathway, p53 pathway, activators of transcription (STAT) pathway, mitogen-activated protein kinase (MAPK) pathway, and stress-activated protein kinase (SAPK) signaling pathway contribute to the activation and regulation of the apoptotic pathway. miRNAs control apoptosis either directly inhibiting expression and translation of proapoptotic 
TABLE 2: Summary of reported miRNAs and their targets in cardiac fibrosis.

\begin{tabular}{|c|c|c|c|c|}
\hline miRNAs & Targets & Effector cells & Signal pathways & References \\
\hline \multicolumn{5}{|c|}{ Antifibrosis } \\
\hline \multirow{2}{*}{ miR-101a } & TGF- $\beta$ R1, & NRCFs and MI rat & TGF $\beta$ signaling & \multirow{2}{*}[47,48]{} \\
\hline & c-Fos & NRCFs and MI rat & MAPK & \\
\hline $\mathrm{miR}-133 / 30$ & CTGF & RCMs, RCFs and Ren2 rat & TGF $\beta$ signaling & [59] \\
\hline miR-24 & Furin & MCFs and MI mouse & TGF $\beta$ signaling & {$[50]$} \\
\hline \multirow{6}{*}{$\operatorname{miR}-29$} & Elastin & RCFs and MI mouse & Protein digestion/absorption & \multirow{6}{*}[61-63]{} \\
\hline & Fbn1 & RCFs and MI mouse & ERK & \\
\hline & Coll $\alpha 1$ & RCFs and MI mouse & ERK & \\
\hline & Coll $\alpha 2$ & RCFs and MI mouse & ERK & \\
\hline & $\operatorname{Col} 3 \alpha 1$ & RCFs and MI mouse & ERK & \\
\hline & FBN, & RCFs and MI mouse & ERK & \\
\hline miR-146a & VEGF & hMSCs & VEGF & {$[65]$} \\
\hline miR-98 & TGF $\beta$ R1 & HCFs & TGF $\beta$ & {$[51]$} \\
\hline \multirow{2}{*}{$\operatorname{miR}-433$} & AZIN1 & & TGF $\beta$ & \multirow{2}{*}[67]{} \\
\hline & JNK1 & & TGF $\beta$ & \\
\hline miR-142-3p & HMGB1 & M6200 cells & $\begin{array}{c}\text { TGF- } \beta 1 / \text { Smad3 signaling } \\
\text { pathway }\end{array}$ & {$[66]$} \\
\hline miR-29b & Tgf $\beta 1$, & MCFs & TGF $\beta$ & {$[64]$} \\
\hline miR-19a-3p/19b-3p & TGF $\beta$ RII & HCFs & TGF $\beta$ signaling & {$[52]$} \\
\hline miR-22 & Smad4 & MCFs & TGF- $\beta$-pathway & {$[58]$} \\
\hline miR-378 & TGF- $\beta 1$ & TAC mouse NMCMs and NRCFs & TGF/Smad signaling & {$[55]$} \\
\hline \multirow{2}{*}{ miR-26a } & COL1, & NRCFs, TAC and miR-26a TG mouse & PI3K-AKT & \multirow[b]{2}{*}[60]{} \\
\hline & CTGF & NRCFs, TAC and miR-26a TG mouse & ECM & \\
\hline \multirow{5}{*}{ miR-15 family } & TGF- $\beta$ R1 & MI mouse and NMCMs & TGF- $\beta$-pathway & \multirow{5}{*}[53,54]{} \\
\hline & p38 & MI mouse and NMCMs & TGF- $\beta$-pathway & \\
\hline & SMAD3 & MI mouse and NMCMs & TGF- $\beta$-pathway & \\
\hline & SMAD7 & MI mouse and NMCMs & TGF- $\beta$-pathway & \\
\hline & Endoglin & MI mouse and NMCMs & TGF- $\beta$-pathway & \\
\hline \multirow{3}{*}{$\operatorname{miR}-21$} & & Profibrosis & & \multirow{3}{*}[56,57]{} \\
\hline & Spryl & NRCFs, NRCMs, TAC and TG mous & ERK-MAPK & \\
\hline & TGF $\beta$ RIII & MI mouse and NRCFs & TGF $\beta 1$-Smad3 signaling & \\
\hline \multirow{3}{*}{ miR-208a } & Endoglin & TAC mouse and RCFs & TGF- $\beta$-pathway & \multirow{3}{*}[28,68,69]{} \\
\hline & b-MHC & TAC mouse and RCFs & TGF- $\beta$-pathway & \\
\hline & THRAP1 & TG mouse & Thyroid hormone & \\
\hline \multirow{7}{*}{$\operatorname{miR}-499$} & Akt & NRCFs & Akt & \multirow{7}{*}[68]{} \\
\hline & MAPKs & NRCFs & MAPK & \\
\hline & Egr1 & NRCFs & ERK & \\
\hline & Egr2 & NRCFs & ERK & \\
\hline & Fos & NRCFs & MAPK & \\
\hline & Myh7 & NRCFs & ERK & \\
\hline & Actal & NRCFs & Smads & \\
\hline miR-122 & TGF- $\beta 1$ & HCFs & TGF $\beta$ & {$[49]$} \\
\hline $\mathrm{miR}-125 \mathrm{~b}$ & Apelin & HCFs, TAC and Ang II induced mouse & TGF $\beta$ & {$[70,71]$} \\
\hline
\end{tabular}

Akt: protein kinase B; $\beta$-MHC: beta myosin heavy chain; c-Fos: proto-oncogene protein; COL1: collagen, type 1; Coll $\alpha 2$ : collagen, type $1 \alpha 2$; Col3 $\alpha 1$ : collagen, type $3 \alpha$ 1; CTGF: connective tissue growth factor; Egrl: early growth response protein 1; Egr2: early growth response protein 2; FBN: fibrillin; Fbn1: fibrillin 1; MAPKs: mitogen-activated protein kinases; p38: tumor protein p38; SMAD3: SMAD family member 3; SMAD2: SMAD family member 2; SMAD7: SMAD family member 7; Spryl: sprouty homolog 1; TGF $\beta$ : transforming growth factor $\beta$; TGF- $\beta 1$ : transforming growth factor $\beta$ 1; TGF- $\beta$ R1: transforming growth factor $\beta$ receptor 1; TGF $\beta$ RIII: transforming growth factor $\beta$ receptor III; THRAP1: mediator complex subunit 13; RCFs: rat cardiac fibroblasts; MCFs: mouse cardiac fibroblasts; HCF: human cardiac fibroblasts; hMSCs: human mesenchymal stem cells; HBMVECs: human brain microvascular endothelial cells. 
molecules or indirectly through regulating the machineries of apoptotic signaling pathways.

Several miRNAs serve as protective factor of cardiomyocyte survival by suppressing/inhibiting the expression of proapoptotic molecules. miR-145 acts on mitochondrial apoptotic pathway by blocking the expression of Bnip3, a mitochondrial apoptosis initiation factor Bnip3. By inhibiting Bnip3 mediated signaling, miR-145 attenuates oxidative stress induced cardiomyocyte cell death caused by $\mathrm{H}_{2} \mathrm{O}_{2}$ or ischemia reperfusion [72]. Interestingly, miR-494 plays vital role in protection against $\mathrm{I} / \mathrm{R}$ injury induced cell death by activating Akt-mitochondrial signaling pathway and antiapoptotic proteins (FGFR2 and LIF) through inhibition of expression of proapoptotic proteins (PTEN, ROCK1, and CaMKII $\delta$ ) [73]. miR-499 also inhibits mitochondrial pathway of cell death by abolishing calcineurin-mediated dephosphorylation of dynamin-related protein-1 (Drpl) and reducing mitochondrial fission [74]. Another research group found that miR-30 is required to prevent abnormal mitochondrial fission associated cell death. However, the downregulation of miR-30 under oxidative stress positively modulates the expression of p53 and Drpl, which consequently leads to cell death [75]. Surprisingly, miR-30 family can target multiple genes of $\beta 1$ and $\beta 2$ adrenergic receptors and $\mathrm{G}$ protein $\alpha$ i subunit (Gi $\alpha 2$ ) to regulate $\beta$-adrenergic signaling pathway [76]. The mitochondrial morphology associated cell death is regulated by miR-181c, which is inversely correlated with the levels of caspases, $\mathrm{Bcl}-2$, and cytochrome $\mathrm{C}$ [77]. In diabetic cardiomyopathy, miR-675 can prevent cardiac apoptosis by suppressing the expression of mitochondrial voltage dependent anion channel 1 (VDAC1), which plays crucial role in activation and release of proapoptotic molecules [78].

Under reticulum stress-associated apoptotic process, the upregulation of miR-185 protects cardiac cells from death by targeting $\mathrm{Na}^{+} / \mathrm{H}^{+}$exchanger-1 (Nhel) [79]. The upregulation of miR-378 enhances cell survival by inhibiting caspase-3 expression in cardiomyocytes during ischemia reperfusion injury [80]. MiR-210 also promotes cardiac cell survival by blocking expression of the negative regulators of VEGF signaling (Efna3 and Ptplb) and apoptosis inducing factor-mitochondrion associated 3 (AIFM3) [81, 82]. Similarly, miR-21 [83] and miR-328 [84] directly target programmed cell death 4 (PDCD4) gene and Atp2a2, respectively. Bim is a Bcl2 family protein, which positively regulates apoptosis by activating caspase cascade of death. Bim expression is inhibited by miR24 [85] and upregulation of miR-24 decreases apoptosis and necrosis in ischemic myocytes by inhibiting BCL2L11 gene [86]. Interestingly, miR-214 prevents $\mathrm{Ca}^{2+}$ overload induced cell death by attenuating several $\mathrm{Ca}^{2+}$ signaling molecules including NCX1, CaMKII $\delta$, CypD, and Bim [87]. In sepsis induced cardiac dysfunction, miR-124 can block cardiomyocyte apoptosis by downregulating the expression of syntaxin-2. However, its expression is suppressed by bacterial LPS under experimental condition [88].

The reduction of blood flow to myocardial tissue produces hypoxia, which imposes oxygen tension and cardiomyocyte cell death. Several miRNAs target hypoxia inducible genes to attenuate or aggravate the oxygen tension induced cardiomyocyte death. miR-199a acts as a master regulator of hypoxia by inhibiting hypoxia inducible factor- $1 \alpha$ (Hif- $1 \alpha$ ) expression and reducing oxygen tension. MiR-199a indirectly abolishes increased level of Hif- $1 \alpha$ by blocking the expression of Sirtl, which is required downregulation of prolyl hydroxylase 2, a molecule involved in stabilization of Hif-1 $\alpha$ [89]. miR-363 controls hypoxia inducible cardiac apoptosis via regulation of Notch1 expression and its downstream signaling [90]. Similarly, the hypoxia induced early expression of miR$146 \mathrm{~b}$ is cardiomyocyte protective by increasing the expression of NF-kB and STAT3, which play cell survival role [91]. miR214 protects cardiomyocyte cell survival and tissue damage caused by hypoxia and I/R injury by suppressing PTEN expression activating PI3K-Akt mediated survival signaling [92]. In contrast, the hypoxia inducible expression of miR200c, miR-92a, and miR-27a promotes cardiomyocyte cell death by targeting GATA-4, Smad 7, and interleukin 10 (IL-10) pathway, respectively [93-95]. GATA4 is a transcription factor required for the cardiomyocyte growth and survival and downregulation of miR-200c promotes GATA-4 dependent expression of antiapoptotic genes such as Bcl2 [93], while inhibition of hypoxia induced increase of miR-92a promotes translation of SMAD7 and blocking NF-kB p65 signaling [94]. miR-138 inhibits expression of Lcn2, a proapoptotic gene by abrogating MLK3/JNK/c-jun signaling pathway [96, 97].

Many miRNAs are upregulated under various pathological conditions in cardiac tissue including myocardial infarction and ischemia/reperfusion injury. They trigger the expression/activity of proapoptotic molecules and cause cardiomyocyte apoptosis. In animal model of ischemia/reperfusion injury, the upregulation of miR-29 inhibits Mcl-2 and enhances Caspase 3 and Bax activity. Interestingly, the activation of peroxisome proliferator-activated receptor (PPAR)$\gamma$ with pioglitazone abrogates proapoptotic effects of miR-29 and protects cardiac cells from death [98]. miR-122 promotes cardiac apoptosis by inhibiting blockers of caspase- 8 and enhancing its expression [99]. Apart from this, miR-378 promotes cardiomyocyte apoptosis by blocking a survival signaling cascade activated by Akt pathway through inhibition of expression of insulin-like growth factor 1 receptor (IGF1R) [100]. Similarly, miR-28 negatively regulates phosphoinositide-dependent kinase-1 (PDK1) and thereby blocks PDK1/Akt/mTOR-dependent signaling of cell survival under oxidative stress condition [101], whereas miR-1 and miR-181c target $\mathrm{Bcl}-2$ expression and favour apoptotic signaling in cardiomyocytes $[75,77]$. In addition, overexpression of miR-1 inhibits the expression of protein kinase $\mathrm{C} \varepsilon(\mathrm{PKC} \varepsilon)$ and heat shock protein 60 (HSP60) and that leads to increased expression of caspase-3 in cardiomyocytes under I/R injury [102]. MiR-195 also downregulates Bcl-2 and Sirtl, which subsequently leads to overproduction of ROS to induce cell death in cardiomyocytes [103]. MiR-15 family members play important role in both cardiomyocyte cell proliferation as well as survival. The expression of miR-15 family inhibits a number of cell cycle genes including checkpoint kinase 1 (Chek1) [104], which causes cell cycle arrest and subsequently cell death. miR-28 [105] and miR-34a [106] block the expression of mitochondrial enzyme aldehyde dehydrogenase 2 


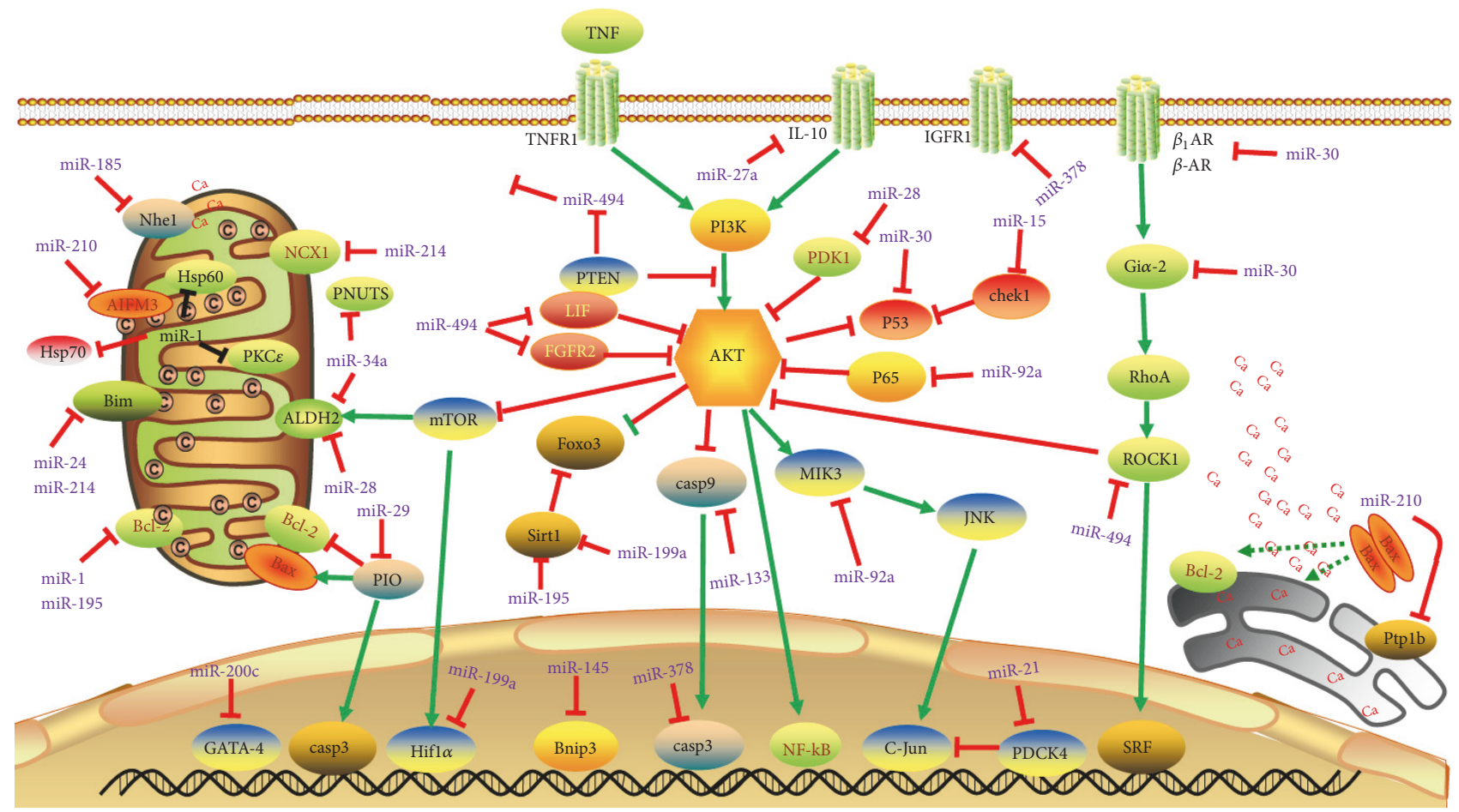

Figure 4: MicroRNAs (miRNAs) in cardiac apoptosis pathways. Arrows colored in red indicate the functions of depression; arrows colored in green indicate the functions of activation. Abbreviations for mRNAs: AIFM3: apoptosis inducing factor; Akt: protein kinase B; ALDH2: aldehyde dehydrogenase 2; Bcl-2: B-cell CLL/lymphoma 2; Bim: Bcl2 like 11; Bnip3: Bcl2/adenovirus E1B 19 kDa interacting protein 3; $\beta 1 \mathrm{AR}$ : adrenoceptor $\beta$ 1; $\beta 2$ AR: adrenoceptor $\beta$ 2; CaMKII $\delta$ : calcium/calmodulin-dependent protein kinase II, $\delta$; Chek1: checkpoint kinase 1; CypD: Cyclophilin+D; FGFR2: fibroblast growth factor receptor 2; Foxo3: Forkhead box O3; Gi $\alpha$ : G protein $\alpha$ i subunit; Hif-1 $\alpha$ : hypoxia inducible factor 1, $\alpha$ subunit; Hsp60: heat shock protein 60; HSP70: heat shock protein 70; IGF-1R: insulin-like growth factor 1 receptor; IL-10: interleukin 10; mTOR: mechanistic target of rapamycin; NCX1: sodium/calcium exchanger 1; Nhel: $\mathrm{Na}^{+} / \mathrm{H}^{+}$exchanger 1; PDCD4: programmed cell death 4; PDK1: 3-phosphoinositide-dependent protein kinase-1; PKCe: protein kinase c betal; PTEN: phosphatase and tensin homolog; Ptplb: Protein Tyrosine Phosphatase-1B; P53: tumor protein p53; p65: tumor protein p65; Rho-A: Ras homolog family member A; ROCK1: Rho associated coiled-coil containing protein kinase 1; SIRT1: Sirtuin 1; TNFR1: tumor necrosis factor receptor superfamily member 1; TNF: tumor necrosis factor; JNK: c-Jun N-terminal kinase; SRF: serum response factor.

(ALDH2), which activates activating AMPK and Akt-mTOR signaling and protecting cardiomyocytes against I/R injury. The inhibition of this pathway aggravates the ischemic injury in myocardial cells [105]. The age related increase of miR-34a aggravates apoptotic cell death in the heart with myocardial injury by suppressing PNUTS and DNA damage responses. This leads to functional decline of the heart [107]. The known miRNAs in cardiac apoptosis and their target genes are summarized in Figure 4 and Table 3.

4.2. miRNA and Autophagy. Autophagy is an evolutionary conserved and highly regulated cellular recycling program that eliminates damaged intracellular organelles as well as injured cells. Emerging studies indicate that many miRNAs affect autophagy by influencing the expression/translation of different machineries involved in the autophagic process (Figure 5). In mammalian system, the autophagy process is initiated by activation of ULKl or ULK2 complexes. The miRNA-17 family members, miRNA-106b and miRNA-20a, block autophagy process by inhibiting the translation of ULKl by targeting the $3^{\prime}$-UTR of ULKl [108]. Experimental studies have shown that miR-25 [109], miR-17-5p [110], miR-4487, and miR-595 [17] can directly target ULK1, while miR-885$3 p$ [111] and miR-26b [112] block autophagy initiation by inhibiting ULK2 [111]. Following the initiation, the vesicle nucleation is one the key processes in autophagy, in which phosphatidylinositol 3-kinase (PI3K) dependent activation and formation of complex composed of VPS15, VPS34, VPS30, and ATG6/BECN1 (Beclin 1) proteins play a central role in vesicle formation. Several miRNAs can modulate the expression of components of above complex and regulate the vesicle nucleation process. miR-30a, miR-384-5p, miR-409$3 p$, and miR-216a directly target the expression of Beclin-1 gene to inhibit vesicle formation [113-117].

The process of vesicle elongation is regulated by two ubiquitin-like protein conjugation systems: Atg5-Atg12-Atg16 protein complex conjugation system and LC3 (microtubuleassociated protein 1 light chain 3 ) conjugation system. Several miRNAs regulate autophagic process by modulating the expression of components of these two ubiquitin-like protein system. Jing et al. found that miRNA-30a/c, miR-18la, miR130a, miR-374a, and miR-630 inhibit the progression of 
TABLE 3: Summary of reported miRNAs and their targets in cardiac apoptosis.

\begin{tabular}{|c|c|c|c|c|}
\hline miRNAs & Targets & Effector cells & Signal pathways & References \\
\hline \multicolumn{5}{|c|}{ Antiapoptosis } \\
\hline miR-145 & Bnip3 & I/R mouse & FOXO & {$[72]$} \\
\hline miR-199a & $\begin{array}{l}\text { Hif- } 1 \alpha, \\
\text { Sirt1 }\end{array}$ & $\begin{array}{l}\text { NRCMs } \\
\text { NRCMs } \\
\end{array}$ & $\begin{array}{l}\text { mTOR } \\
\text { AT1R }\end{array}$ & [89] \\
\hline miR-21 & PDCD4 & NRCMs & NF-kB & [83] \\
\hline miR-328 & Atp2a2 & H9C2 cells & & {$[84]$} \\
\hline miR-214 & $\begin{array}{l}\text { NCX1 } \\
\text { PTEN }\end{array}$ & $\begin{array}{l}\text { NRCM and miR-214 KO mouse } \\
\text { I/R mouse }\end{array}$ & $\begin{array}{l}\text { Calcium signaling } \\
\text { PI3K-AKT } \\
\end{array}$ & $\begin{array}{l}{[87]} \\
{[92]}\end{array}$ \\
\hline miR-24 & Bim & NMCMs & Mitochondrial apoptosis & {$[85,86]$} \\
\hline miR-146b & RNase L & H9c2 cells & NF-kB & [91] \\
\hline miR-378 & Caspase-3 & H9c2 cells and AMI rat & MAPK & {$[80]$} \\
\hline miR-494 & $\begin{array}{l}\text { PTEN } \\
\text { ROCK1 } \\
\text { CaMKII } \delta \\
\text { FGFR2 } \\
\text { LIF }\end{array}$ & $\begin{array}{l}\text { miR-494 TG Mouse } \\
\text { miR-494 TG Mouse } \\
\text { miR-494 TG Mouse } \\
\text { miR-494 TG Mouse } \\
\text { miR-494 TG Mouse }\end{array}$ & $\begin{array}{l}\text { PI3K-AKT } \\
\text { cGMP-PKG } \\
\text { HIF1 } \\
\text { PI3K-AKT } \\
\text { TNF } \\
\end{array}$ & {$[73]$} \\
\hline miR-499 & Drp1 & Male C57BL/6 mice & Mitochondrial pathway & {$[74]$} \\
\hline miR-185 & Nhe1 & NRVMs & cAMP & [79] \\
\hline miR-30 family & $\begin{array}{l}\beta 1 \mathrm{AR}, \\
\beta 2 \mathrm{AR}, \\
\text { Gi } \alpha 2 \\
\text { P53 } \\
\text { Drp1 }\end{array}$ & $\begin{array}{l}\text { MI rat, DOX-induced HF rat, ARCM and H9c2 cells } \\
\text { MI rat, DOX-induced HF rat, ARCM and H9c2 cells } \\
\text { MI rat, DOX-induced HF rat, ARCM and H9c2 cells } \\
\text { NRCMs } \\
\text { NRCMs }\end{array}$ & $\begin{array}{l}\beta \text {-adrenergic pathway } \\
\beta \text {-adrenergic pathway } \\
\beta \text {-adrenergic pathway } \\
\text { p53 } \\
\text { mitochondrial pathway }\end{array}$ & {$[75,76]$} \\
\hline miR-210 & $\begin{array}{l}\text { Efna3, } \\
\text { Ptplb } \\
\text { AIFM3 }\end{array}$ & $\begin{array}{l}\text { Mouse HL-1 cardiomyocytes and adult FVB mice } \\
\text { Mouse HL-1 cardiomyocytes, adult FVB mice } \\
\text { H9c2 cells, neonatal rat cardiomyocytes (NRCM) }\end{array}$ & $\begin{array}{c}\text { VEGF signaling } \\
\text { VEGF signaling } \\
\text { Mitochondrial apoptosis } \\
\end{array}$ & {$[81,82]$} \\
\hline miR-92a & NF-kB p65 & rat H9c2 cells & NF-kB & {$[94]$} \\
\hline miR-675 & VDAC1 & DCM & Mitochondrial apoptosis & {$[78]$} \\
\hline miR-138 & $\operatorname{Lcn} 2$ & HL-1 cells & Mitochondrial apoptosis & {$[96,97]$} \\
\hline miR-124 & STX2 & Male BALB/c rats & miR-124a/STX2 pathway & [88] \\
\hline \multicolumn{5}{|c|}{ Proapoptosis } \\
\hline miR-1 & $\begin{array}{l}\mathrm{HSP} 60 \\
\mathrm{Bcl}-2 \\
\mathrm{PKC} \varepsilon\end{array}$ & $\begin{array}{l}\text { LNA-antimiR-1 treated mouse } \\
\text { H9c2 cells and I/R rat } \\
\text { LNA-antimiR-1 treated mouse }\end{array}$ & $\begin{array}{l}\text { RNA degradation } \\
\text { Mitochondrial apoptosis } \\
\text { cGMP-PKG }\end{array}$ & {$[75,102]$} \\
\hline miR-200c & GATA-4 & NMCMs & cGMP-PKG & [93] \\
\hline miR-363 & Notch1 & Rat H9C2 cell & Notch signaling & {$[90]$} \\
\hline miR-122 & caspase- 8 & NMCMs & ERK-MAPK & [99] \\
\hline miR-181c & $\mathrm{Bcl}-2$ & NMCMs & Mitochondrial apoptosis & {$[77]$} \\
\hline miR-15 & Chek1 & C57BL/6 mice and TG mouse & & {$[104]$} \\
\hline miR-34a & $\begin{array}{l}\text { PNUTS } \\
\text { ALDH2 }\end{array}$ & $\begin{array}{l}\text { NRCMs and MI rat } \\
\text { NRCMs and MI rat }\end{array}$ & $\begin{array}{l}\text { Oxidative stress } \\
\text { Oxidative stress }\end{array}$ & {$[106,107]$} \\
\hline miR-378 & IGF1R & NMCMs and H9C2 cells & MAPK & {$[100]$} \\
\hline miR-27a & IL-10 & Sprague-Dawley rats and H9c2 cell & Interleukin 10 (IL-10) pathway & {$[95]$} \\
\hline miR-29 & $\mathrm{PIO}$ & H9c2 cells & PPAR & {$[98]$} \\
\hline miR-28 & $\begin{array}{c}\text { PDK1 } \\
\text { ALDH2 }\end{array}$ & $\begin{array}{l}\text { NMCMs } \\
\text { NMCMs }\end{array}$ & $\begin{array}{l}\text { PDK1/Akt/mTOR-dependent signaling } \\
\text { AMPK and Akt-mTOR signaling. }\end{array}$ & {$[101,105]$} \\
\hline miR-195 & $\begin{array}{l}\text { Sirt1 } \\
\text { Bcl-2 }\end{array}$ & $\begin{array}{l}\text { C57BL/ } 6 \text { mouse and NMCMs } \\
\text { C57BL/6 mouse and NMCMs }\end{array}$ & $\begin{array}{c}\text { AMPK } \\
\text { Mitochondrial apoptosis }\end{array}$ & {$[103]$} \\
\hline
\end{tabular}

AIFM3: apoptosis inducing factor; ALDH2: aldehyde dehydrogenase 2; Bcl-2: B-cell CLL/lymphoma 2; Bim: Bcl2 like 11; Bnip3: Bcl2/adenovirus E1B 19 kDa interacting protein 3; $\beta 1 \mathrm{AR}$ : adrenoceptor $\beta$ 1; $\beta 2 \mathrm{AR}$ : adrenoceptor $\beta$ 2; CaMKII $\delta$ : calcium/calmodulin-dependent protein kinase II, $\delta$; Chek1: checkpoint kinase 1; CypD: Cyclophilin+D; Drp1: dynamin-related protein-1; Efna3: ephrin A3; FGFR1: fibroblast growth factor receptor 1; Gi $\alpha 2$ : G protein $\alpha$ i subunit; Hif$1 \alpha$ : hypoxia-inducible factor 1, $\alpha$ subunit; HSP60: heat shock protein 60; IGF-1R: insulin-like growth factor 1 receptor; IL-10: interleukin 10; Lcn2: lipocalin-2; LIF: leukemia inhibitory factor; NCX1: sodium/calcium exchanger 1; Nhel: Na+/H+ exchanger 1; PDCD4: programmed cell death 4; PDK1: 3-phosphoinositidedependent protein kinase-1; PKCe: protein kinase c betal; PTEN: phosphatase and tensin homolog; Ptplb: Protein Tyrosine Phosphatase-1B; P53: tumor protein p53; p65: tumor protein p65; ROCK1: Rho associated coiled-coil containing protein kinase 1; SIRT1: Sirtuin 1; SMAD7: SMAD family member 7; DCM: diabetic cardiomyopathy. 


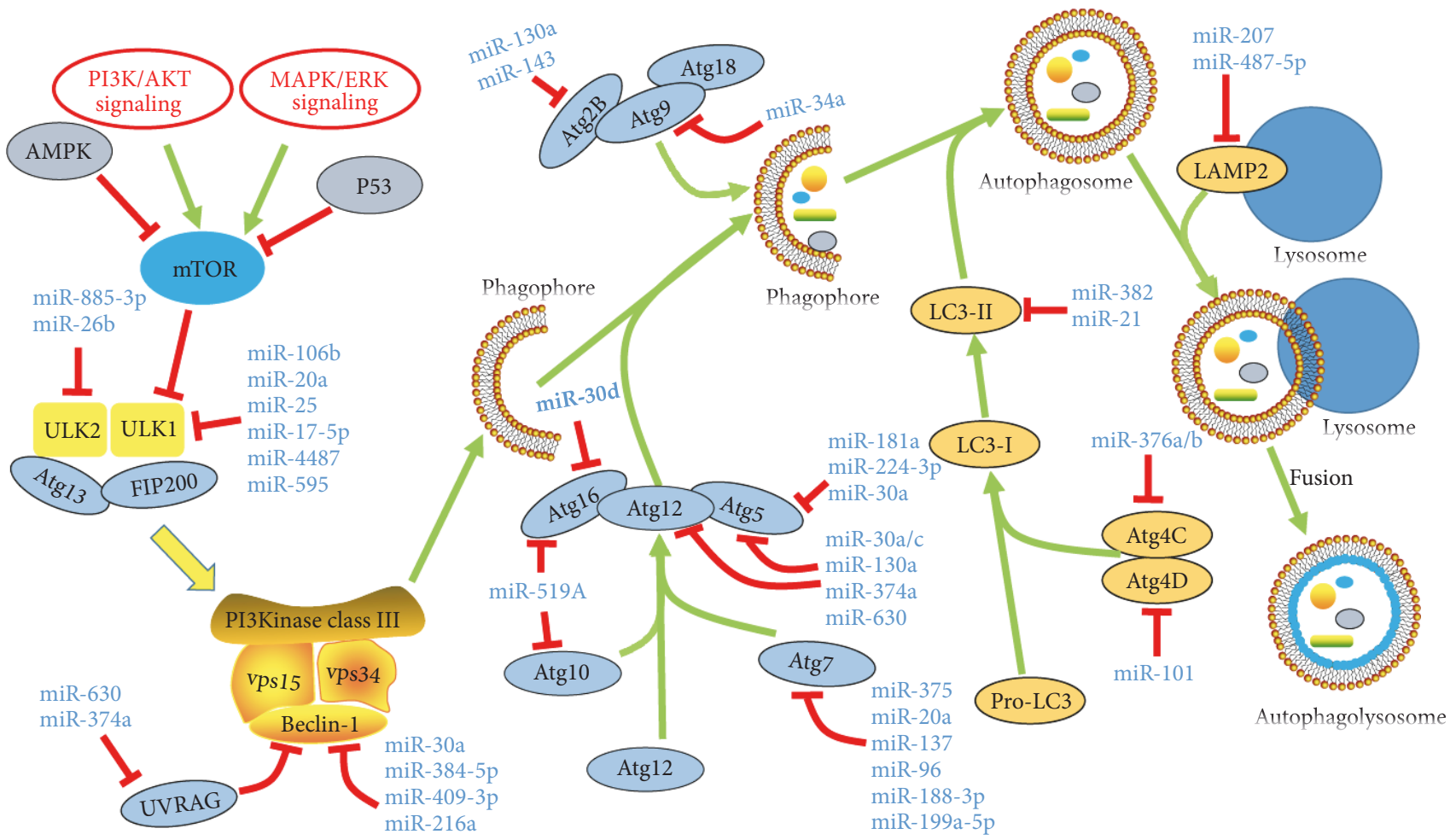

FIGURE 5: miRNAs in cardiac Autophagy pathways. Abbreviations for mRNAs: AMPK: adenosine 5' -monophosphate- (AMP-) activated protein kinase; ATG: AuTophaGy; UVRAG: UV radiation resistance associated gene; VSP: venom serine protease; LAMP2: lysosomal associated membrane protein 2 .

autophagic process by inhibiting expression of Atg5 and Atg12 [191], whereas miR-181a [119], miR-30a [121], and miR224-3p [120] only interact with Atg5 mRNA resulting in reduction of its translation. In addition, miR-30d directly targets Atg16 to block the formation of Atg5-Atg12-Atg16 protein complex [122]. miR-519A has the capability to suppress expression of both Atg16 and Atg10 [118]. Several research groups found that miR-375, miR-20a, miR-137, miR96, miR-188-3p, and miR-199a-5p block autophagic process by directly targeting Atg7, an autophagic protein involved in the formation of Atg7-Atg10 complex [32, 123-127]. Atg4 family is involved in the processing of LC3. miR-376 family members such as miR-376a and miR-376b inhibit translation of Atg4C and attenuate autophagic process [128, 129]. miR101 suppresses expression of Atg4D abd blocks Lc3 processing [130]. Wang et al. [131] found that silencing of endogenous miR-382 function in renal mesangial cells can induce LC3-related autophagy. Ursolic acid is shown to improve autophagy and increase the expression of LC3-II to attenuate diabetic mesangial cell injury through attenuated miRNA21/PTEN/Akt/mTOR signal pathway [132].

Apart from them, MiR-130a and miR-143 interfere the formation of Atg9-Atg2-Atg18 complex by targeting Atg2B and inhibiting autophagosome assembly [133, 134]. Another research group found that miRNA-34a attenuates autophagic process by downregulating the expression of Atg9 gene [135]. The fusion of autophagosome-lysosome fusion is mediated by key autophagic proteins such as RAB1B, RAB22A, RAB14,
RAB27A, LAMP2, and LAMP3. Many research groups found that miR-502 and miR-451 inhibit RAB1B and RAB14, respectively $[136,137]$. LAMP2 can be suppressed by miR-207 and miR-487-5p, while miR-205 blocks RAB27A and LAMP3 and interfering autophagic vesicle maturation process $[138,140$, 192]. The two other miRNAs such as miR-630 and miR-374a can directly target UVRAG and thus affect the maturation of autophagosomes [118]. The known miRNAs in autophagy and their target genes are illustrated in Figure 5 and Table 4.

4.3. miRNA, Inflammatory Response, and Pyroptosis. The activation and accumulation of immune cells such as macrophage are of the earliest responses to cardiac tissue injury that governs the clearance of cell debris, stimulation of compensatory growth and cardiac tissue regeneration. The macrophage polarization and activation are the key process involved in the determination of the inflammatory response, cardiac tissue injury, angiogenesis, scar formation, and cardiac tissue remodeling [139]. In general, the activation of classical M1 macrophage at early phase initiates inflammatory response by increased production of proinflammatory cytokines and chemokines. However, the polarization of macrophages generates alternative M2 macrophage phenotype, which exerts inflammation suppression, apoptotic cell clearance, and tissue repair [139]. miRNAs modulate the activity of macrophages and they can regulate the expression of several proinflammatory cytokines. miR-155 plays multiple roles in the inflammatory process. miR-155 regulates 
TABLE 4: Summary of reported miRNAs and their targets in Autophagy.

\begin{tabular}{|c|c|c|c|}
\hline miRNAs & Targets & Effector cells (Tissue) & References \\
\hline \multicolumn{4}{|c|}{ miRNAs regulate the induction of autophagy } \\
\hline miR-106b & ULKl & C2C12 (myoblast cells) & [108] \\
\hline miR-25 & ULKl & MCF-7 (breast cancer) & [109] \\
\hline miR-17-5p & ULKl & TIB-71 (murine macrophage cells) & [110] \\
\hline $\operatorname{miR}-4487$ & ULKl & SH-SY5Y (neuroblastoma) & {$[17]$} \\
\hline miR-595 & ULKl & SH-SY5Y (neuroblastoma) & {$[17]$} \\
\hline miR-885-3p & ULK2 & JHU-029 (squamous cell carcinoma) & [111] \\
\hline miR-26b & ULK2 & PC3, C4-2 (prostate cancer) & [112] \\
\hline \multicolumn{4}{|c|}{ miRNAs regulate the vesicle nucleation of autophagy } \\
\hline miR-30a & Beclin-1 & 786-0, A489 (renal carcinoma), MG-63 (osteosarcoma) & {$[113,114]$} \\
\hline miR-384-5p & Beclin-1 & primary mouse macrophages & [115] \\
\hline $\operatorname{miR}-409-3 p$ & Beclin-1 & Lovo Oxa R (colorectal cancer) & [116] \\
\hline $\operatorname{miR}-216 a$ & Beclin-1 & PANC-1 (pancreas cancer) & [117] \\
\hline $\operatorname{miR}-630$ & UVRAG & JHU-029 (squamous cell carcinoma) & [118] \\
\hline miR-374a & UVRAG & JHU-029 (squamous cell carcinoma) & [118] \\
\hline \multicolumn{4}{|c|}{ miRNAs regulate the vesicle elongation and retrieval of autophagy } \\
\hline miR-181a & Atg5 & MCF-7 (breast cancer) Huh7 (liver cancer) K562 (chronic myelogenous leukemia) & {$[119]$} \\
\hline miR-224-3p & Atg5 & U251 and U87 (glioblastoma) & {$[120]$} \\
\hline miR-30a & Atg5 & K562 (CML) & {$[121]$} \\
\hline miR-30d & Atg16 & A2780, OVCAR10 and 2008 (ovarian cancer), T47D and MCF-7 (breast cancer) & [122] \\
\hline miR-375 & ATG7 & Huh7, Hep3B (liver cancer) & [123] \\
\hline miR-20a & ATG7 & $\mathrm{SiHa}$ (cervical cancer) & [124] \\
\hline miR-137 & ATG7 & U87 (glioblastoma) & [125] \\
\hline miR-96 & ATG7 & LNCaP, 22Rv1, and LAPC4 (prostate cancer) & {$[32]$} \\
\hline miR-188-3p & ATG7 & NMCMs & [126] \\
\hline miR-199a-5p & ATG7 & Huh7, HepG2 (liver cancer) & [127] \\
\hline miR-519A & ATG16, ATG10 & JHU-029 (squamous cell carcinoma) & [118] \\
\hline $\mathrm{miR}-376 \mathrm{a} / \mathrm{b}$ & ATG4C & MCF-7 (breast cancer) Huh7 (liver cancer) & {$[128,129]$} \\
\hline miR-101 & ATG4D & MCF-7 (breast cancer) & {$[130]$} \\
\hline miR-382 & LC3 & renal mesangial cells & {$[131]$} \\
\hline miR-21 & LC3-II & diabetic mesangial cell & {$[132]$} \\
\hline MiR-130a & $\operatorname{Atg} 2 B$ & MEC-1 (leukemia) & {$[133]$} \\
\hline miR-143 & Atg2B & H1299 (lung cancer) & [134] \\
\hline miR-34a & Atg9 & NMCMs & [135] \\
\hline \multicolumn{4}{|c|}{ miRNAs regulate the formation and degradation of autolysosome } \\
\hline miR-502 & RAB1B & HCT116 (colorectal cancer) & [136] \\
\hline $\operatorname{miR}-451$ & RAB14 & A549, SPC-A1, and NCI-H520 (lung cancer) & [137] \\
\hline miR-207 & LAMP2 & primary cortical neuronal cells & [138] \\
\hline miR-487-5p & LAMP2 & A549, H1299 (lung cancer) & [139] \\
\hline miR-205 & RAB27A, LAMP3 & DU145, PC3 (prostate cancer) & [140] \\
\hline
\end{tabular}

AMPK: adenosine $5^{\prime}$-monophosphate- (AMP-) activated protein kinase; mTOR: mammalian target of rapamycin; ATG: AuTophaGy; UVRAG: UV radiation resistance associated gene; VSP: venom serine protease; LAMP2: lysosomal associated membrane protein 2; NMCMs: neonatal mouse cardiomyocytes; RAB1B: member RAS oncogene family; RAB14: member RAS oncogene family; RAB27A: member RAS oncogene family; LAMP3: lysosomal associated membrane protein 3.

macrophage polarization by controlling SOCS1 and Akt1 axis [141]. In viral myocarditis, the increased expression of miR-155 blocks macrophage polarization and alternatively activated M2 macrophages phenotype transformation. This results in increased pathogen induced inflammation and cardiac injury [142]. miR-155 also promotes macrophage survival by upregulating the SHIP1-Akt signaling cascade [143]. The increased level of macrophage derived miR-155 acts as paracrine regulator of cardiac fibroblast proliferation as well as inflammatory response following the myocardial infarction induced injury [144]. In contrast, another research group found that miR-155 functions as a negative feedback 
TABLE 5: Summary of reported miRNAs and their targets in Inflammatory Response.

\begin{tabular}{lccc}
\hline miRNAs & Targets & Regulation factors & References \\
\hline MiR-155 & SOCS1, SHIP1, IL-13Ra1 & IFN-b, TNF-a, IL-1 & LPS \\
miR-125a & TNFAIP3 (A20) & LPS & [141-146] \\
miR-125b & TNFAIP3, TNF-a & LPS & [147] \\
miR-125a-5p & TLR2, TLR4 & LPS & {$[148]$} \\
miR-21 & CSF-1R & PPARg & {$[149]$} \\
miR-22 & Rasal, Nfat5 & M-CSF & {$[150]$} \\
miR-27a & PPARg & M-CSF \\
Mir-375 & PDK1 & LPS & {$[151]$} \\
MiR-126 & ADAM9 & [152] \\
MiR-145-5p & CD40 & IL-1 $\beta$, TNF- $\alpha$, IL-6 & {$[153]$} \\
MiR-146a & IRAK, TRAF6 & LPS, TNF- $\alpha$ & {$[154]$} \\
\hline
\end{tabular}

SOCS1: suppressor of cytokine signaling 1; SHIP1: inositol polyphosphate-5-phosphatase D; TNF-a: tumor necrosis factor a; LPS: lipopolysaccharides; CSF-1R: colony stimulating factor 1 receptor; Rasal: RAS p21 protein activator 1; Nfat5: nuclear factor of activated T-cells 5; PDK1: pyruvate dehydrogenase kinase 1; IRAK: interleukin 1 receptor associated kinase; TRAF6: TNF receptor associated factor 6; ADAM9: A disintegrin and metalloproteinase 9.

regulator of immune response by reducing the expression of cytokines by cardiac myocytes during viral infection induced myocarditis [145]. Similarly, miR-155 can block the activity of macrophages by directly targeting proinflammatory NF$\kappa \mathrm{B}$ signaling transcription factor in atherosclerotic plaque [146]. Apart from this, the colony stimulating factor 1 (CSF-1) dependent expression of miR-21 positively regulates macrophage polarization. It promotes amplification of M2 macrophage phenotype and directly targets proinflammatory molecules [149]. PPAR $\gamma$ promotes alternative activation and polarization of macrophage (M2) by enhancing the expression of miR-22, which targets Rasal and Nfat5 [150]. However, miR-27a switches on M1 macrophage polarization by blocking PPAR $\gamma$ [151]. mir-375 promotes inflammation induced cardiomyocyte cell death by inhibiting PDK1-Akt signaling and increasing proinflammatory cytokines [152].

miR-125 family is considered as a therapeutic target for the prevention of inflammation. miR-125a and miR-125b directly suppress the expression of tumor necrosis factor alpha-induced protein 3 (TNFAIP3, A20), which is a negative regulator of NF-KB signaling [147]. Another research group found that TLR2 and TLR4 dependent upregulation of miR$125 \mathrm{a}-5 \mathrm{p}$ suppresses classical pathway of macrophage activation and inflammation. However, this miR-125a-5p stimulates alternative pathways of macrophage polarization and activation [148]. Interestingly, miR-125b has been reported to suppress the activity and stability of TNF- $\alpha$ transcript and thereby it reduces inflammatory responses [123]. Efferocytosis is a process of the clearance of apoptotic cardiomyocyte by macrophages. This process is vital for clear resolution of inflammatory response and tissue repair after cardiac injury. miR-126 promotes the efferocytosis in myocardial tissue. However, the reduction of its expression under diabetic condition impairs the clearance of dead cardiomyocytes, which consequently leads to prolonged inflammation process [153]. Similarly, miR-145-5p can inhibit the CD40 mediated inflammatory response and cardiomyocyte cell death caused by acute hypoxia. But its expression is downregulated under severe hypoxia condition [154]. In sepsis induced cardiac injury and dysfunction, miR-146a can attenuate NF$\mathrm{kB}$ dependent inflammatory signaling by targeting IRAK and TRAF6 in cardiomyocytes and monocytes [155].

Pyroptosis is a proinflammatory form of cell death, which morphologically resembles apoptotic and necrotic mode of cell death. This mode of cell death is triggered by the activation of inflammasome, which leads to the release of inflammatory cytokines. Caspase-1 is the major effector molecule involved in this form of cell death [193]. miR-30d promotes cardiomyocyte pyroptosis under diabetic cardiomyopathy by repressing Foxo3a and its downstream molecule apoptosis repressor with caspase recruitment domain (ARC), which consequently leads to upregulation of the expression of caspase 1 and proinflammatory cytokines (IL-1 and IL-18) in diabetic cardiomyopathy [64]. In opposite to this, miR-9 inhibits inflammatory cytokine secretion and TNF $\alpha$ induced pyroptosis by inhibiting the expression of ELAVlike protein 1 (ELAVL1) and this inhibition reduces the expression of NLRP3, caspase-1, and IL-1 $\beta$ [194]. Bauernfeind et al. found that miR-223 negatively regulates NLRP3 inflammasome activity and this inhibition could block the initiation of pyroptosis [195]. Currently, very few reports are available to directly link miRNAs and pyroptosis in cardiac tissue. However, their role in the immune cell activation and inflammatory cytokine expression indicates that more $\mathrm{miR}$ would be involved in the regulation of pyroptosis process. The known miRNAs in inflammatory response and their target genes are summarized in Table 5.

\section{5. miRNA and Angiogenesis}

Neovascularization is the process of new blood vessel formation, which is generated from vascular endothelial cells (EC). This process is essential for the blood supply and nutrients to the recovered part of tissues after various injury [196]. The vascular regeneration is one of the important 
pathological features of ventricular remodeling. In pressure overload myocardial hypertrophy, the growth rate of vascular tissue is less than the processes of cardiomyocyte hypertrophy. This leads to a significant decrease in the density and causes formation of thin myocardial blood vessels, which relatively reduces blood supply to the heart and eventually affecting heart function. Emerging evidences reveal that miRNAs play fundamental role in maintaining the vascular integrity, endothelial cell proliferation, migration, blood vessel formation and sprouting in the adult heart in response to injury. Under hypoxia and vascular injury condition, the upregulation of miR-126 activates endothelial cells (EC) and endothelial progenitor cells (EPCs) in order to promote vascular healing and vessel formation. The endothelial specific expression of miR-126 promotes the development and maintenance of vessels by enhancing the expression of angiogenic factors such as VEGF and FGF. This is achieved by directly suppressing Spred-land phosphoinositol-3 kinase regulatory subunit 2 (PIK3R2/p85-b), which is a intracellular antiangiogenic signaling [156]. MiR-130a contributes to angiogenesis by suppressing antiangiogenic homeobox gene GAX and HOXA5 in endothelial cells. In addition, miR-130a activates PI3K/Akt dependent signaling by inhibiting PTEN expression and that attenuates remodeling after myocardial infarction induced injury $[158,159]$. The miR-17-92 cluster is highly expressed in human endothelial cells. This cluster expresses six miRNAs (miR-17, miR-18a, miR-19a, miR20a, miR-19b-1, and miR-92a-1), which are transcriptionally regulated by c-Myc [160]. The miRNAs such as miR-494 and miR-126-5p act as proangiogenic factors by targeting bone morphogenetic protein 4 (BMP4) [197], while miR18a downregulates CTGF expression in response to Myc activation and promotes angiogenesis [198, 199]. MR-19a primarily targets angiogenesis-inhibitor, thrombospondin-1 (Tsp1) in vascular system. Likewise, the hypoxia induced upregulation of miR-210 promotes endothelial cell migration and capillary like structure formation by targeting Receptor Tyrosine Kinase Ligand Ephrin-A3 and PTP1b [81, 161, 162]. miR-126 also contributes to vascular angiogenesis through targeting Cysteine-rich 61 (CCN1) in PTEN/Akt pathway [157]. miR-146a promotes angiogenesis by increasing the expression of VEGF and reducing the fibrotic process at injury site [65].

The growth factors upregulate miR-296 and this upregulation favours angiogenesis by reducing levels of hepatocyte growth factor-regulated tyrosine kinase substrate (HGS) through targeting HGS mRNA, which is responsible for degradation of angiogenic receptors such as VEGFR2 and PDGFRb in endothelial cells [163]. Sonic hedgehog (Shh) signaling is involved in the formation of large-diameter vessel by inducing expression of angiogenic cytokines, including VEGF and angiopoietin-1 (Ang-1) and Ang-2 (Ang-2). It is well known that suppressor of fused $(\mathrm{SuFu})$ is a negative regulator Shh signaling. The upregulation of miR-378 promotes cell survival, tumor growth, and angiogenesis by suppressing $\mathrm{SuFu}$ and Fus-1 expression [164]. In addition, increased level of miR-378 expression stimulates endothelial cell angiogenic activity [165]. In experimental animal model, the endothelial expression of miR-132 displayed angiogenic potential by suppressing endothelial p120RasGAP expression and promoting Ras activation, which is required for the induction of neovascularization [166]. miR-23 and miR27 promote angiogenesis by inhibiting two antiangiogenic factors such as Sprouty2 and Sema6A proteins [167], while miR-27b expression accelerates angiogenesis by regulating translation of Dll4/Notch axis, PPAR $\gamma$ and its downstream effectors [168]. Some miRNAs play distinct role in MI heart. For instance, miR-24 has displayed that different activities depend on the cell types in cardiac tissue. In the postinfarct heart, miR-24 enhances survival, proliferation, and angiogenic function of EC by targeting GATA2, p21-activated kinase PAK4, and eNOS. However, it has detrimental role on cardiomyocytes and fibroblasts by inducing cell death $[169,170]$.

Several miRNAs oppose the angiogenic process in endothelial cells. miR-214 prevents cardiac angiogenesis by reducing the expression of X-box binding protein 1 (XBP1); an important transcription factor contributes to endothelial cells proliferation and tube formation [171]. In response to stress, the upregulation of miR-34 family members (miR-34a, miR-34b, and miR-34c) [172], in particular, miR-34a, stimulates endothelial progenitor cell senescence and impedes its angiogenic activity through attenuation of the expression of silent information regulator 1 (SIRT1) [173, 174]. In animal model of MI, silencing of miR-34 family attenuates MI-induced pathological left ventricular remodeling after MI and improves cardiac function by suppressing vascular endothelial growth factors (VEGF), vinculin, protein Ofucosyltransferase 1 (Pofut1), Notch1, and semaphorin $4 \mathrm{~B}$ (Sema4b) [172]. By targeting the proangiogenesis TGFb-1, miR-29a and miR-101a inhibit TGF $\beta$ pathway in endothelial cells in MI rats [175]. Overexpression of miR-15b-5p suppresses arteriogenesis and angiogenesis in mice by directly targeting protein kinase B-3 (AKT3) [176]. Some miRNAs play distinct role in MI heart. For instance, miR-24 has displayed that different activities depend on the cell types in cardiac tissue. In the postinfarct heart, miR-24 enhances survival, proliferation, and angiogenic function of EC by targeting GATA2, p21-activated kinase PAK4, and eNOS. However, it has detrimental role in cardiomyocytes and fibroblasts by inducing cell death $[169,170]$.

miR-100 has antiangiogenic function by blocking mTOR signaling in endothelial and vascular smooth muscle cells, and its inhibition promotes the regulation of revascularization [177]. EMT is one of the major mechanisms for the development and progression of fibrosis. It is well known that ZEB1/SIP1 is a suppressor of E-cadherin and upregulation of ZEB1/SIP leads to the loss of epithelial phenotype. Surprisingly, miR-200b and ZEB1/SIP1 have a feedback loop regulation to control epithelial-mesenchymal transition (EMT) and increased expression of miR-200b can suppress ZEB1/SIP1 during EMT [178, 179]. In endothelial cells, miR-200b regulates angiogenic signals by targeting VEGF signaling receptors including VEGFR2, Flt1, KDR, and GATA binding protein 2 (GATA2) [180, 181].

Several other miRNAs such as miR-15a, miR-16, and miR-424 target FGF and VEGF signaling components and 


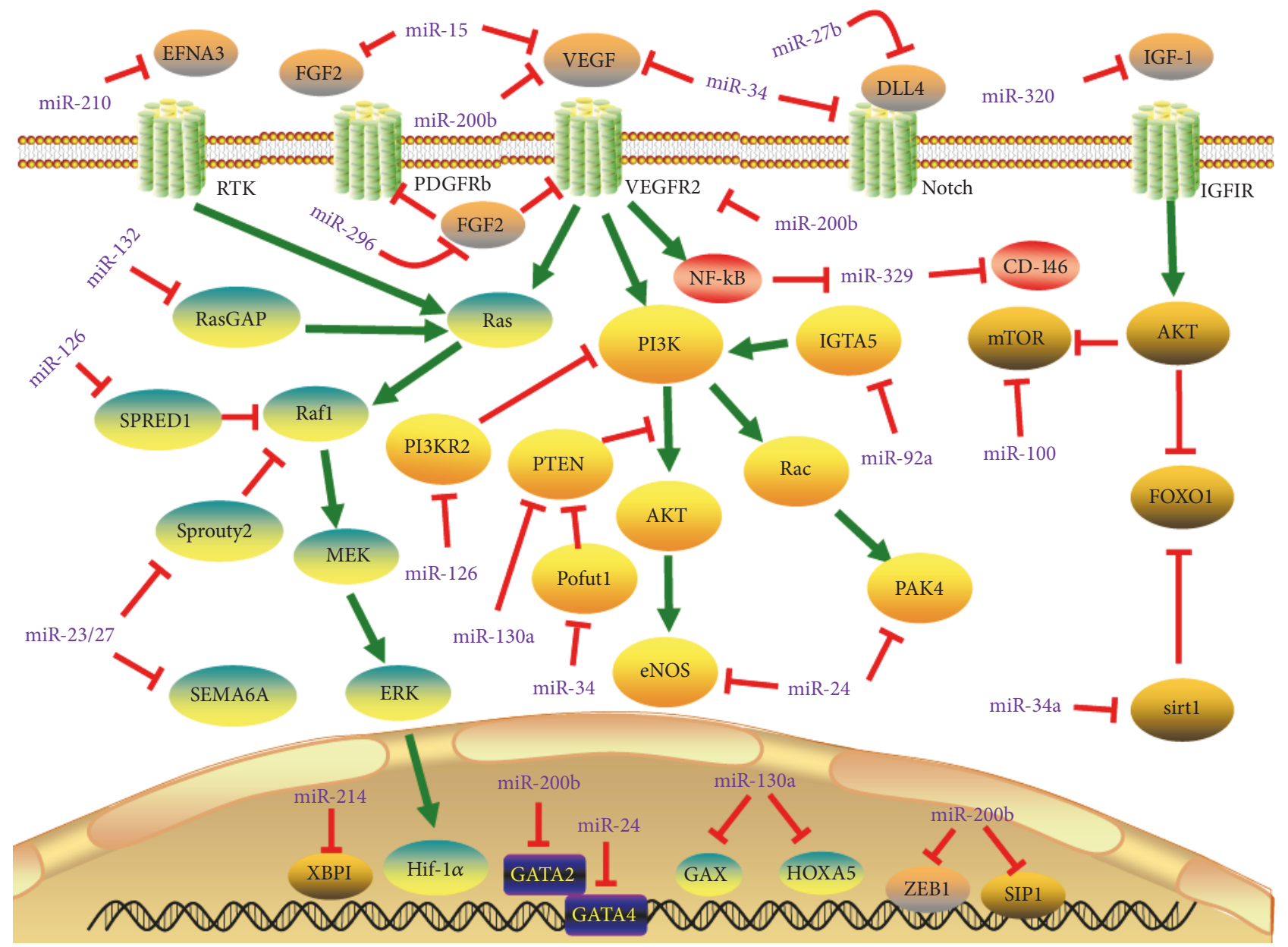

FIGURE 6: MicroRNAs (miRNAs) in angiogenesis pathways. Arrows colored in red indicate the functions of depression; arrows colored in green indicate the functions of activation. Abbreviations for Dll4: delta-like protein 4; Efna3: ephrin A3; eNOS: endothelial nitric oxide synthase; FGF2: fibroblast growth factor 2; Foxo1: Forkhead box O1; GATA2: GATA binding protein 2; Gata4: GATA binding protein 4; GAX: gaseous oxygen; HGS: hepatocyte growth factor-regulated tyrosine kinase substrate; Hif-1 $\alpha$ : hypoxia inducible factor 1, $\alpha$ subunit; HOXA5: homeobox A5; IGF-1: insulin-like growth factor 1; IGF-1R: insulin-like growth factor 1 receptor; mTOR: mechanistic target of rapamycin; PIK3R2: phosphoinositol-3 kinase regulatory subunit 2; PTEN: phosphatase and tensin homolog; RasGAP: Ras GTPase-activating protein; Sema6A: semaphorin 6A; SIP1: Smad interacting protein 1; SIRT1: Sirtuin 1; Sprouty2: sprouty homolog 2; VEGF: vascular endothelial growth factor; VEGFR2: vascular endothelial growth factor receptor 1; XBP1: X-box binding protein 1; ZEB1: zinc finger E-box-binding homeobox 1; RTK: receptor tyrosine kinase; Casp9, caspase 9; Casp3, caspase 3; ERK: extracellular regulated protein kinases; Spread-1: Sprouty-related, EVH1 domain-containing protein 1; PDGFR $\beta$ : platelet-derived growth factor receptor $\beta$.

impose antiangiogenic effects. MiR-15a suppresses FGF2 and VEGF expression, but miR-16 and miR-424 block VEGFR2 and FGFR1 receptor expression in endothelial cells [183, 184]. When the glucose availability is high and growth factor level is low, the endothelial expression of miR-503 is increased, which causes impairment of endothelial function and angiogenesis via directly inhibiting molecules involved in cell proliferation and survival proteins such as cdc25A and CCNE1 [182]. The upregulation of miR-320 expression suppresses insulin-like growth factor-1 (IGF-1) activity in myocardial microvascular endothelial cells and thus impaired angiogenesis in diabetic condition [185]. Astonishingly, the expression of miR-320 by cardiomyocytes controls endothelial function under diabetic condition. In experimental diabetic animals, miR-320 is transferred from cardiomyocytes to endothelial cells by exosomal activity and this transfer regulates endothelial cells migration and tube formation by downregulating IGF-1, Hsp20, and Ets2 [186]. miR-329 abrogates angiogenesis by targeting CD146, an endothelial adhesion molecule required to maintain endothelial cell integrity [187]. In cardiac ischemic condition, miR-92a targets integrin subunits a5 (ITGA5) and causes blockage on angiogenesis, which worsens the ischemic injury [188, 189]. The suppression of miR-92a with antagonist after ischemia reperfusion injury improves the functional recovery of damaged tissue by promoting blood vessel growth [190]. The known miRNAs involved in angiogenesis and their target genes are summarized in Figure 6 and Table 6. 
TABLE 6: Summary of reported miRNAs and their targets in angiogenesis.

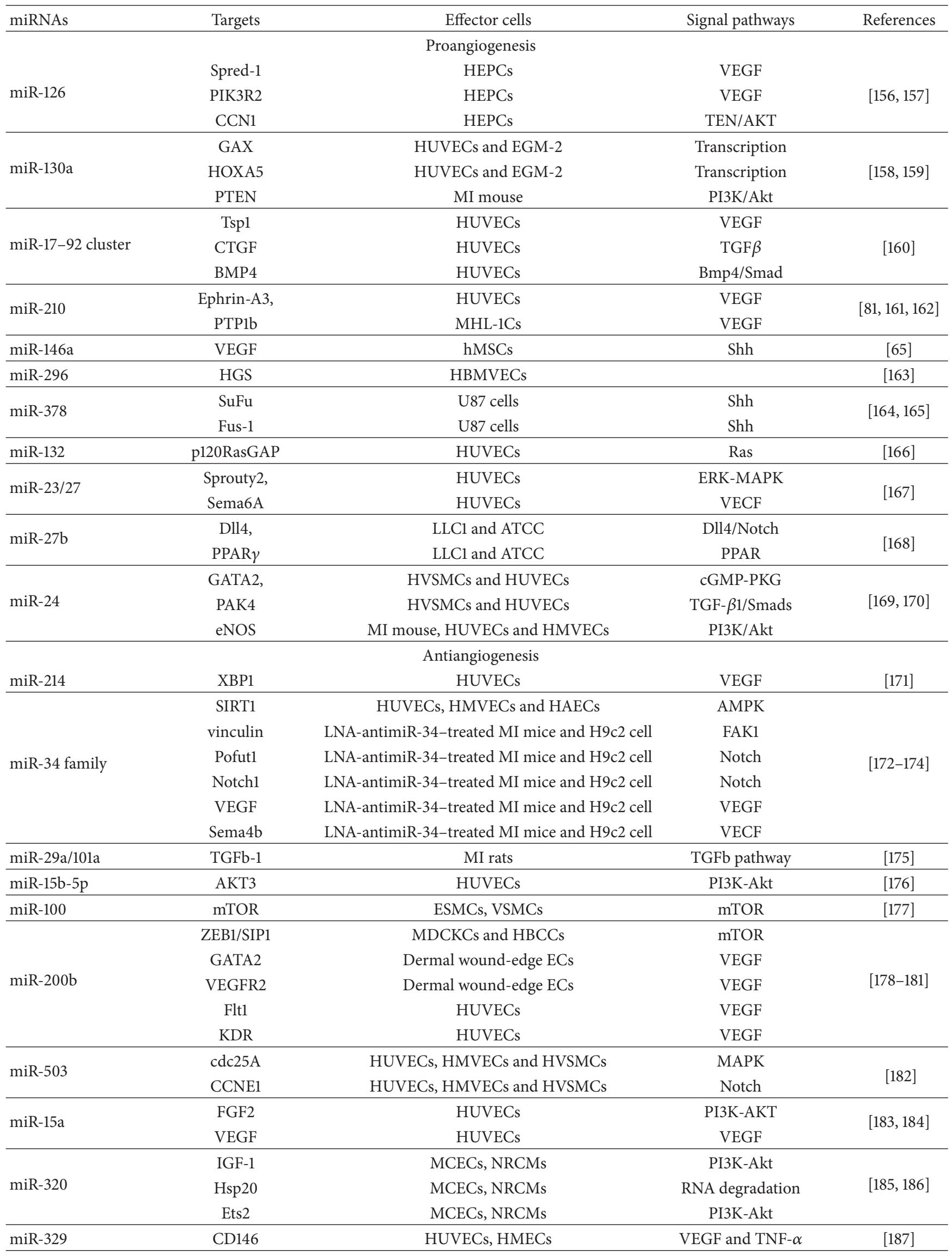


TABLE 6: Continued.

\begin{tabular}{llccc}
\hline miRNAs & Targets & Effector cells & Signal pathways & References \\
\hline miR-92a & ITGA5 & RMUG-S, OVISE and RMG-1 & Rho/ROCK & {$[188-190]$} \\
\hline
\end{tabular}

CCNE1: cyclin E1; cdc25A: cell division cycle 25A; CTGF: connective tissue growth factor; Dll4: delta-like protein 4; eNOS: endothelial nitric oxide synthase; Ets2: Euro Truck Simulator 2; FGF2: fibroblast growth factor 2; FGFR1: fibroblast growth factor receptor 1; GATA2: GATA binding protein 2; GAX: gaseous oxygen; HGS: hepatocyte growth factor-regulated tyrosine kinase substrate; HOXA5: homeobox A5; IGF-1: insulin-like growth factor 1; ITGA5: integrin subunits a5; mTOR: mechanistic target of rapamycin; PIK3R2: phosphoinositol-3 kinase regulatory subunit 2; Pofut1: protein O-fucosyltransferase 1; Ppary: peroxisome proliferator-activated receptor $\gamma$; PTEN: phosphatase and tensin homolog; Sema4b: semaphorin 4B; Sema6A: semaphorin 6A; SIP1: Smad interacting protein 1; SIRT1: Sirtuin 1; Sprouty2: sprouty homolog 2; Tsp1: thrombospondin-1; VEGF: vascular endothelial growth factor; VEGFR2: vascular endothelial growth factor receptor 1; XBP1: X-box binding protein 1; ZEB1: zinc finger E-box-binding homeobox 1; FAK: focal adhesion kinase; HUVECs: human umbilical vein ECs; EGM-2: endothelial growth medium 2; MHL-1Cs: mouse HL-1 cardiomyocytes; hMSCs: human mesenchymal stem cells; Shh: sonic hedgehog signaling; HBMVECs: human brain microvascular endothelial cells; LLC1: Lewis lung carcinoma; ATCC: RAW 264.7 mouse macrophage cell lines; HVSMCs: human vascular smooth muscle cells; HUVECs: human umbilical vein endothelial cells; HMVECs: human microvascular ECs; HAECs: human aortic endothelial cell; ESMCs: endothelial smooth muscle cells; VSMCs: vascular smooth muscle cells; HVSMCs: human VSMCs cells; MDCKCs: Madin-Darby canine kidney cells; HBCCs: human breast cancer cells; dermal wound-edge ECs: dermal wound-edge endothelial cells; MCECs: mouse cardiac endothelial cells; NRCMs: neonatal rat cardiomyocytes; HMECs: human microvascular endothelial cells.

\section{Conclusion}

Myocardial remodeling due to various insults to the heart is a multifactorial and complex process. To prevent and manage the cardiac dysfunction due to postinjury related cardiac remodeling, it is important to have a clear understanding about the occurrence, development, and progression of cardiac remodeling in the injured heart. Numerous research findings in recent years suggest that miRNAs have unavoidable role in cardiac structure remodeling by differentially regulating thousands of different mRNAs. Many miRNAs have been proposed as prognosis factors and therapeutic tools for myocardial remodeling. Thus, miRNA-based strategies can be a promising approach to target network of myocardium remodeling related genes. In recent years, the pharmacological development of miRNA inhibitors/activators such as miR-mimics, antagomiRs, and decoys to manipulate miRNAs expression level has made a significant progress to utilize them as therapeutic tools for cardiac failure caused by myocardial remodeling. In experimental models mimicking human heart diseases, the exogenous administration of miRNA inhibitors or miRNA mimics can alleviate the remodeling process and improve cardiovascular disease. However, specificity, half-life of these molecules, efficiency, and route of administration are the major challenge to bring them from the workbench to the bedside. By solving these issues by joint efforts of basic researchers and clinical scientist, miRNA could be better and most effective therapeutic regimen to manage cardiovascular problems associated with cardiac remodeling.

\section{Conflicts of Interest}

The authors declare no conflicts of interest.

\section{Acknowledgments}

This work was supported by the China Postdoctoral Science Foundation Funded Projects (2016M592133) and (2016M592132); Qingdao Postdoctoral Application Research Funded Project (2016069); the National Science Foundation for Distinguished Young Scholars of China (81600244).

\section{References}

[1] R. Dash, V. J. Kadambi, A. G. Schmidt et al., "Interactions between phospholamban and $\beta$-adrenergic drive may lead to cardiomyopathy and early mortality," Circulation, vol. 103, no. 6, pp. 889-896, 2001.

[2] R. C. Lee, R. L. Feinbaum, and V. Ambros, "The C. elegans heterochronic gene lin-4 encodes small RNAs with antisense complementarity to lin-14," Cell, vol. 75, no. 5, pp. 843-854, 1993.

[3] E. Lund, S. Güttinger, A. Calado, J. E. Dahlberg, and U. Kutay, "Nuclear export of microRNA precursors," Science, vol. 303, no. 5654, pp. 95-98, 2004.

[4] Y. Lee, K. Jeon, J.-T. Lee, S. Kim, and V. N. Kim, "MicroRNA maturation: stepwise processing and subcellular localization," The EMBO Journal, vol. 21, no. 17, pp. 4663-4670, 2002.

[5] T. A. McKinsey and E. N. Olson, "Toward transcriptional therapies for the failing heart: Chemical screens to modulate genes," Journal of Clinical Investigation, vol. 115, no. 3, pp. 538546, 2005.

[6] E. van Rooij, W. S. Marshall, and E. N. Olson, "Toward microRNA-based therapeutics for heart disease: the sense in antisense," Circulation Research, vol. 103, no. 9, pp. 919-928, 2008.

[7] S. Ikeda, A. He, S. W. Kong et al., "MicroRNA-1 negatively regulates expression of the hypertrophy-associated calmodulin and Mef2a genes," Molecular and Cellular Biology, vol. 29, no. 8, pp. 2193-2204, 2009.

[8] G. P. Diniz, C. A. Lino, C. R. Moreno, N. Senger, and M. L. M. Barreto-Chaves, "MicroRNA-1 overexpression blunts cardiomyocyte hypertrophy elicited by thyroid hormone," Journal of Cellular Physiology, 2017.

[9] M. He, Z. Yang, M. Abdellatif, and D. Sayed, "GTPase activating protein (SH3 domain) binding protein 1 regulates the processing of microRNA-1 during cardiac hypertrophy," PLoS ONE, vol. 10, no. 12, Article ID e0145112, 2015.

[10] F. Varrone, B. Gargano, P. Carullo et al., "The circulating level of FABP3 is an indirect biomarker of microRNA-1," Journal of the American College of Cardiology, vol. 61, no. 1, pp. 88-95, 2013.

[11] I. Karakikes, A. H. Chaanine, S. Kang et al., "Therapeutic cardiac-targeted delivery of miR-1 reverses pressure overloadinduced cardiac hypertrophy and attenuates pathological remodeling.", Journal of the American Heart Association, vol. 2, no. 2, p. e000078, 2013.

[12] H. Zhang, J. Wu, H. Dong, S. A. Khan, M.-L. Chu, and T. Tsuda, "Fibulin-2 deficiency attenuates angiotensin II-induced 
cardiac hypertrophy by reducing transforming growth factor- $\beta$ signalling," Clinical Science, vol. 126, no. 4, pp. 275-288, 2014.

[13] G. P. Diniz, C. A. Lino, E. C. Guedes, L. D. Nascimento Moreira, and M. L. M. Barreto-Chaves, "Cardiac microRNA-133 is downregulated in thyroid hormone-mediated cardiac hypertrophy partially via Type 1 Angiotensin II receptor," Basic Research in Cardiology, vol. 110, no. 5, article 49, 2015.

[14] A. Carè, D. Catalucci, F. Felicetti et al., "MicroRNA-133 controls cardiac hypertrophy," Nature Medicine, vol. 13, no. 5, pp. 613618, 2007.

[15] D. Wang, G. Zhai, Y. Ji, and H. Jing, "microRNA-10a targets T-box 5 to inhibit the development of cardiac hypertrophy," International Heart Journal, vol. 58, no. 1, pp. 100-106, 2017.

[16] Y. Xiao, X. Zhang, S. Fan, G. Cui, and Z. Shen, "MicroRNA-497 inhibits cardiac hypertrophy by targeting Sirt4," PLoS ONE, vol. 11, no. 12, Article ID e0168078, 2016.

[17] Y.-S. Wang, J. Zhou, K. Hong, X.-S. Cheng, and Y.-G. Li, "MicroRNA-223 displays a protective role against cardiomyocyte hypertrophy by targeting cardiac troponin I-interacting kinase," Cellular Physiology and Biochemistry, vol. 35, no. 4, pp. 1546-1556, 2015.

[18] C. Wu, S. Dong, and Y. Li, "Effects of miRNA-455 on cardiac hypertrophy induced by pressure overload," International Journal of Molecular Medicine, vol. 35, no. 4, pp. 893-900, 2015.

[19] J. Ganesan, D. Ramanujam, Y. Sassi et al., "MiR-378 controls cardiac hypertrophy by combined repression of mitogen-activated protein kinase pathway factors," Circulation, vol. 127, no. 21, pp. 2097-2106, 2013.

[20] R. S. Nagalingam, N. R. Sundaresan, M. P. Gupta, D. L. Geenen, R. J. Solaro, and M. Gupta, "A cardiac-enriched microRNA, miR-378, blocks cardiac hypertrophy by targeting Ras signaling," The Journal of Biological Chemistry, vol. 288, no. 16, pp. 11216-11232, 2013.

[21] R. Li, G. Yan, Q. Zhang et al., "miR-145 inhibits isoproterenolinduced cardiomyocyte hypertrophy by targeting the expression and localization of GATA6," FEBS Letters, vol. 587, no. 12, pp. 1754-1761, 2013.

[22] Y. Duan, B. Zhou, H. Su, Y. Liu, and C. Du, "MiR-150 regulates high glucose-induced cardiomyocyte hypertrophy by targeting the transcriptional co-activator p300," Experimental Cell Research, vol. 319, no. 3, pp. 173-184, 2013.

[23] Y. Yang, Y. Zhou, Z. Cao et al., "miR-155 functions downstream of angiotensin II receptor subtype 1 and calcineurin to regulate cardiac hypertrophy," Experimental and Therapeutic Medicine, vol. 12, no. 3, pp. 1556-1562, 2016.

[24] A. Ucar, S. K. Gupta, J. Fiedler et al., "The miRNA-212/132 family regulates both cardiac hypertrophy and cardiomyocyte autophagy," Nature Communications, vol. 3, article 1078, 2012.

[25] K. Wang, Z.-Q. Lin, B. Long, J.-H. Li, J. Zhou, and P.-F. Li, "Cardiac hypertrophy is positively regulated by microRNA miR-23a," Journal of Biological Chemistry, vol. 287, no. 1, pp. 589-599, 2012.

[26] M. Li, N. Wang, J. Zhang et al., "MicroRNA-29a-3p attenuates ET-1-induced hypertrophic responses in H9c2 cardiomyocytes," Gene, vol. 585, no. 1, pp. 44-50, 2016.

[27] G. P. Diniz, A. P. Takano, and M. L. M. Barreto-Chaves, "MiRNA-208a and miRNA-208b are triggered in thyroid hormone-induced cardiac hypertrophy-role of type 1 Angiotensin II receptor (AT1R) on miRNA-208a/ $\alpha$-MHC modulation," Molecular and Cellular Endocrinology, vol. 374, no. 1-2, pp. 117-124, 2013.
[28] E. van Rooij, L. B. Sutherland, X. Qi, J. A. Richardson, J. Hill, and E. N. Olson, "Control of stress-dependent cardiac growth and gene expression by a microRNA," Science, vol. 316, no. 5824, pp. 575-579, 2007.

[29] Q. Bao, L. Chen, J. Li et al., "Role of microRNA-124 in cardiomyocyte hypertrophy inducedby angiotensin II," Cellular and Molecular Biology, vol. 63, no. 4, pp. 23-27, 2017.

[30] Q. Bao, M. Zhao, L. Chen et al., "MicroRNA-297 promotes cardiomyocyte hypertrophy via targeting sigma-1 receptor," Life Sciences, vol. 175, pp. 1-10, 2017.

[31] J. Shi, Y. Bei, X. Kong et al., "miR-17-3p contributes to exercise-induced cardiac growth and protects against myocardial ischemia-reperfusion injury," Theranostics, vol. 7, no. 3, pp. 664-676, 2017.

[32] C. Bang, S. Batkai, and S. Dangwal, "Cardiac fibroblast-derived microRNA passenger strand-enriched exosomes mediate cardiomyocyte hypertrophy," The Journal of Clinical Investigation, vol. 124, no. 5, pp. 2136-2146, 2014.

[33] H. Y. Seok, J. Chen, M. Kataoka et al., "Loss of MicroRNA155 protects the heart from pathological cardiac hypertrophy," Circulation Research, vol. 114, no. 10, pp. 1585-1595, 2014.

[34] X.-D. Xu, X.-W. Song, Q. Li, G.-K. Wang, Q. Jing, and Y.-W. Qin, "Attenuation of microRNA-22 derepressed PTEN to effectively protect rat cardiomyocytes from hypertrophy," Journal of Cellular Physiology, vol. 227, no. 4, pp. 1391-1398, 2012.

[35] Z.-P. Huang, J. Chen, H. Y. Seok et al., "MicroRNA-22 regulates cardiac hypertrophy and remodeling in response to stress," Circulation Research, vol. 112, no. 9, pp. 1234-1243, 2013.

[36] J. Yang, Y. Nie, F. Wang et al., "Reciprocal regulation of miR-23a and lysophosphatidic acid receptor signaling in cardiomyocyte hypertrophy," Biochimica et Biophysica Acta-Molecular and Cell Biology of Lipids, vol. 1831, no. 8, pp. 1386-1394, 2013.

[37] X.-Y. You, J.-H. Huang, B. Liu, S.-J. Liu, Y. Zhong, and S.-M. Liu, "HMGA1 is a new target of miR-195 involving isoprenalineinduced cardiomyocyte hypertrophy," Biochemistry, vol. 79, no. 6, pp. 538-544, 2014.

[38] D. Zheng, J. Ma, Y. Yu et al., "Silencing of miR-195 reduces diabetic cardiomyopathy in C57BL/6 mice," Diabetologia, vol. 58, no. 8, pp. 1949-1958, 2015.

[39] D. W. Song, J. Y. Ryu, J. O. Kim, E. J. Kwon, and D. H. Kim, "The miR-19a/b family positively regulates cardiomyocyte hypertrophy by targeting atrogin-1 and MuRF-1," Biochemical Journal, vol. 457, no. 1, pp. 151-162, 2014.

[40] J. Wang, Y. Song, Y. Zhang et al., "Cardiomyocyte overexpression of miR-27b induces cardiac hypertrophy and dysfunction in mice," Cell Research, vol. 22, no. 3, pp. 516-527, 2012.

[41] C. Li, X. Li, X. Gao et al., "MicroRNA-328 as a regulator of cardiac hypertrophy," International Journal of Cardiology, vol. 173, no. 2, pp. 268-276, 2014.

[42] J. O. Kim, D. W. Song, E. J. Kwon et al., "MiR-185 plays an anti-hypertrophic role in the heart via multiple targets in the calcium-signaling pathways," PLoS ONE, vol. 10, no. 3, Article ID e0122509, 2015.

[43] L. Wei, M. Yuan, R. Zhou et al., "MicroRNA-101 inhibits rat cardiac hypertrophy by targeting Rabla," Journal of Cardiovascular Pharmacology, vol. 65, no. 4, pp. 357-363, 2015.

[44] R. L. Montgomery, T. G. Hullinger, H. M. Semus et al., "Therapeutic inhibition of miR-208a improves cardiac function and survival during heart failure," Circulation, vol. 124, no. 14, pp. 1537-1547, 2011. 
[45] A. Deb and E. Ubil, "Cardiac fibroblast in development and wound healing," Journal of Molecular and Cellular Cardiology, vol. 70, pp. 47-55, 2014.

[46] S. N. Cilvik, J. I. Wang, K. J. Lavine et al., "Fibroblast growth factor receptor 1 signaling in adult cardiomyocytes increases contractility and results in a hypertrophic cardiomyopathy," PLoS ONE, vol. 8, no. 12, Article ID e82979, 2013.

[47] Z. Pan, X. Sun, H. Shan et al., "MicroRNA-101 inhibited postinfarct cardiac fibrosis and improved left ventricular compliance via the FBJ osteosarcoma oncogene/transforming growth factor- $\beta 1$ pathway," Circulation, vol. 126 , no. 7, pp. 840-850, 2012.

[48] X. Zhao, K. Wang, Y. Liao et al., "MicroRNA-101a inhibits cardiac fibrosis induced by hypoxia via targeting TGF $\beta$ RI on cardiac fibroblasts," Cellular Physiology and Biochemistry, vol. 35, no. 1, pp. 213-226, 2015.

[49] J. Beaumont, B. López, N. Hermida et al., "microRNA-122 down-regulation may play a role in severe myocardial fibrosis in human aortic stenosis through TGF- $\beta 1$ up-regulation," Clinical Science, vol. 126, no. 7, pp. 497-506, 2014.

[50] J. Wang, W. Huang, R. Xu et al., "MicroRNA-24 regulates cardiac fibrosis after myocardial infarction," Journal of Cellular and Molecular Medicine, vol. 16, no. 9, pp. 2150-2160, 2012.

[51] R. Cheng, R. Dang, Y. Zhou, M. Ding, and H. Hua, "MicroRNA98 inhibits TGF- $\beta 1$-induced differentiation and collagen production of cardiac fibroblasts by targeting TGFBR1," Human Cell, vol. 30, pp. 1-9, 2017.

[52] M. Zou, F. Wang, R. Gao et al., "Autophagy inhibition of hsamiR-19a-3p/19b-3p by targeting TGF- $\beta$ R II during TGF- $\beta 1-$ induced fibrogenesis in human cardiac fibroblasts," Scientific Reports, vol. 6, Article ID 24747, 2016.

[53] T. G. Hullinger, R. L. Montgomery, and A. G. Seto, "Inhibition of miR-15 protects against cardiac ischemic injury," Circulation Research, vol. 110, pp. 71-81, 2012.

[54] E. R. Porrello, A. I. Mahmoud, E. Simpson et al., "Regulation of neonatal and adult mammalian heart regeneration by the miR15 family," Proceedings of the National Academy of Sciences of the United States of America, vol. 110, no. 1, pp. 187-192, 2013.

[55] R. S. Nagalingam, N. R. Sundaresan, M. Noor, M. P. Gupta, R. J. Solaro, and M. Gupta, "Deficiency of cardiomyocyte-specific MicroRNA-378 contributes to the development of cardiac fibrosis involving a transforming growth factor $\beta$ (TGF $\beta 1$ )dependent paracrine mechanism," Journal of Biological Chemistry, vol. 289, no. 39, pp. 27199-27214, 2014.

[56] H. Liang, C. Zhang, T. Ban et al., "A novel reciprocal loop between microRNA-21 and TGF $\beta$ RIII is involved in cardiac fibrosis," International Journal of Biochemistry and Cell Biology, vol. 44, no. 12, pp. 2152-2160, 2012.

[57] D. Zhang, Y. Cui, B. Li, X. Luo, B. Li, and Y. Tang, "miR-155 regulates high glucose-induced cardiac fibrosis via the TGF- $\beta$ signaling pathway," Molecular BioSystems, vol. 13, no. 1, pp. 215224, 2017.

[58] Y. Huang, Y. Qi, J.-Q. Du, and D.-F. Zhang, "MicroRNA34a regulates cardiac fibrosis after myocardial infarction by targeting Smad4," Expert Opinion on Therapeutic Targets, vol. 18, no. 12, pp. 1355-1365, 2014.

[59] R. F. Duisters, A. J. Tijsen, B. Schroen et al., "MiR-133 and miR30 Regulate connective tissue growth factor: implications for a role of micrornas in myocardial matrix remodeling," Circulation Research, vol. 104, no. 2, pp. 170-178, 2009.
[60] C. Wei, I.-K. Kim, S. Kumar et al., "NF- $\kappa$ B mediated miR-26a regulation in cardiac fibrosis," Journal of Cellular Physiology, vol. 228, no. 7, pp. 1433-1442, 2013.

[61] G. M. Manzoni, G. Castelnuovo, and R. Proietti, "Assessment of psychosocial risk factors is missing in the 2010 ACCF/AHA guideline for assessment of cardiovascular risk in asymptomatic adults," Journal of the American College of Cardiology, vol. 57, no. 14, pp. 1569-1570, 2011.

[62] E. Van Rooij, L. B. Sutherland, J. E. Thatcher et al., "Dysregulation of microRNAs after myocardial infarction reveals a role of miR-29 in cardiac fibrosis," Proceedings of the National Academy of Sciences of the United States of America, vol. 105, no. 35, pp. 13027-13032, 2008.

[63] K. Dawson, R. Wakili, B. Ördög et al., "MicroRNA29: a mechanistic contributor and potential biomarker in atrial fibrillation," Circulation, vol. 127, no. 14, pp. 1466-1475, 2013.

[64] X. Li, N. Du, and Q. Zhang, "MicroRNA-30d regulates cardiomyocyte pyroptosis by directly targeting foxo3a in diabetic cardiomyopathy," Cell Death and Disease, vol. 5, no. 10, Article ID e1479, 2014.

[65] H. Seo, S. Lee, C. Y. Lee et al., "Exogenous mirna-146a enhances the therapeutic efficacy of human mesenchymal stem cells by increasing vascular endothelial growth factor secretion in the ischemia/reperfusion-injured heart," Journal of Vascular Research, vol. 54, no. 2, pp. 100-108, 2017.

[66] J. Y. Y. Ooi, B. C. Bernardo, and J. R. McMullen, “Therapeutic potential of targeting microRNAs to regulate cardiac fibrosis: MiR-433 a new fibrotic player," Annals of Translational Medicine, vol. 4, no. 24, article no. 548, 2016.

[67] Y. Wang, M. Ouyang, Q. Wang, and Z. Jian, "MicroRNA-142-3p inhibits hypoxia/reoxygenation-induced apoptosis and fibrosis of cardiomyocytes by targeting high mobility group box 1," International Journal of Molecular Medicine, vol. 38, no. 5, pp. 1377-1386, 2016.

[68] Y. Huang and J. Li, "MicroRNA208 family in cardiovascular diseases: therapeutic implication and potential biomarker," Journal of Physiology and Biochemistry, vol. 71, no. 3, pp. 479486, 2015.

[69] K.-G. Shyu, B.-W. Wang, W.-P. Cheng, and H.-M. Lo, "MicroRNA-208a increases myocardial endoglin expression and myocardial fibrosis in acute myocardial infarction," Canadian Journal of Cardiology, vol. 31, no. 5, pp. 679-690, 2015.

[70] V. Nagpal, R. Rai, A. T. Place et al., "MiR-125b is critical for fibroblast-to-myofibroblast transition and cardiac fibrosis," Circulation, vol. 133, no. 3, pp. 291-301, 2015.

[71] K. Siddiquee, J. Hampton, S. Khan et al., "Apelin protects against angiotensin II-induced cardiovascular fibrosis and decreases plasminogen activator inhibitor type-1 production," Journal of Hypertension, vol. 29, no. 4, pp. 724-731, 2011.

[72] R. Li, G. Yan, Q. Li et al., "MicroRNA-145 protects cardiomyocytes against hydrogen peroxide (H2O2)-induced apoptosis through targeting the mitochondria apoptotic pathway," PLoS ONE, vol. 7, no. 9, Article ID e44907, 2012.

[73] X. Wang, X. Zhang, X.-P. Ren et al., "MicroRNA-494 targeting both proapoptotic and antiapoptotic proteins protects against ischemia/reperfusion-induced cardiac injury," Circulation, vol. 122, no. 13, pp. 1308-1318, 2010.

[74] Y.-C. Zhao, "Effects of exercise training on myocardial mitochondrial miR-499-CaN-Drp-1 apoptotic pathway in mice," Chinese Journal of Applied Physiology, vol. 31, no. 3, pp. 259-263, 2015. 
[75] J. Li, S. Donath, Y. Li, D. Qin, B. S. Prabhakar, and P. Li, "miR-30 regulates mitochondrial fission through targeting p53 and the dynamin-related protein-1 pathway," PLoS Genetics, vol. 6, no. 1, Article ID e1000795, 2010.

[76] L. Roca-Alonso, L. Castellano, A. Mills et al., "Myocardial MiR30 downregulation triggered by doxorubicin drives alterations in $\beta$-adrenergic signaling and enhances apoptosis," Cell Death and Disease, vol. 6, no. 5, Article ID e1754, 2015.

[77] H. Wang, J. Li, H. Chi et al., "MicroRNA-181c targets Bcl-2 and regulates mitochondrial morphology in myocardial cells," Journal of Cellular and Molecular Medicine, vol. 19, no. 9, pp. 2084-2097, 2015.

[78] X. Li, H. Wang, B. Yao, W. Xu, J. Chen, and X. Zhou, "LncRNA H19/miR-675 axis regulates cardiomyocyte apoptosis by targeting VDAC1 in diabetic cardiomyopathy," Scientific Reports, vol. 6, Article ID 36340, 2016.

[79] J. O. Kim, E. J. Kwon, D. W. Song, J. S. Lee, and D. H. Kim, “miR185 inhibits endoplasmic reticulum stress-induced apoptosis by targeting $\mathrm{Na}+/ \mathrm{H}+$ exchanger-1 in the heart," BMB Reports, vol. 49, no. 4, pp. 208-213, 2016.

[80] J. Fang, X.-W. Song, J. Tian et al., "Overexpression of microRNA-378 attenuates ischemia-induced apoptosis by inhibiting caspase- 3 expression in cardiac myocytes," Apoptosis, vol. 17, no. 4, pp. 410-423, 2012.

[81] S. Hu, M. Huang, Z. Li et al., "MicroRNA-210 as a novel therapy for treatment of ischemic heart disease," Circulation, vol. 122, no. 11, pp. S124-S131, 2010.

[82] R. K. Mutharasan, V. Nagpal, Y. Ichikawa, and H. Ardehali, "microRNA-210 is upregulated in hypoxic cardiomyocytes through Akt- and p53-dependent pathways and exerts cytoprotective effects," American Journal of Physiology-Heart and Circulatory Physiology, vol. 301, no. 4, pp. H1519-H1530, 2011.

[83] J. Xiao, Y. Pan, X. H. Li et al., "Cardiac progenitor cell-derived exosomes prevent cardiomyocytes apoptosis through exosomal miR-21 by targeting PDCD4," Cell Death \& Disease, vol. 7, no. 6, Article ID e2277, 2016.

[84] X. Zheng, X. Hu, T. Ge et al., "MicroRNA-328 is involved in the effect of selenium on hydrogen peroxide-induced injury in H9c2 cells," Journal of Biochemical and Molecular Toxicology, vol. 31, no. 8, p. e21920, 2017.

[85] L. Qian, L. W. Van Laake, Y. Huang, S. Liu, M. F. Wendland, and D. Srivastava, "miR-24 inhibits apoptosis and represses Bim in mouse cardiomyocytes," The Journal of Experimental Medicine, vol. 208, no. 3, pp. 549-560, 2011.

[86] D. F. Li, J. Tian, X. Guo et al., "Induction of microrna-24 by hif-1 protects against ischemic injury in rat cardiomyocytes," Physiological Research, vol. 61, pp. 555-565, 2012.

[87] A. B. Aurora, A. I. Mahmoud, X. Luo et al., "MicroRNA-214 protects the mouse heart from ischemic injury by controlling $\mathrm{Ca}^{2+}$ overload and cell death," Journal of Clinical Investigation, vol. 122, no. 4, pp. 1222-1232, 2012.

[88] X. Diao and S. Sun, "PMicroRNA-124a regulates LPS-induced septic cardiac dysfunction by targeting STX2," Biotechnology Letters, vol. 39, no. 9, pp. 1335-1342, 2017.

[89] S. Rane, M. He, D. Sayed et al., "Downregulation of MiR199a derepresses hypoxia-inducible factor- $1 \alpha$ and sirtuin 1 and recapitulates hypoxia preconditioning in cardiac myocytes," Circulation Research, vol. 104, no. 7, pp. 879-886, 2009.

[90] X. Meng, Y. Ji, Z. Wan et al., "Inhibition of miR-363 protects cardiomyocytes against hypoxia-induced apoptosis through regulation of Notch signaling," Biomedicine and Pharmacotherapy, vol. 90, pp. 509-516, 2017.
[91] J.-W. Li, S.-Y. He, Z.-Z. Feng et al., "MicroRNA-146b inhibition augments hypoxia-induced cardiomyocyte apoptosis," Molecular Medicine Reports, vol. 12, no. 5, pp. 6903-6910, 2015.

[92] X. Wang, T. Ha, Y. Hu et al., "MicroRNA-214 protects against hypoxia/reoxygenation induced cell damage and myocardial ischemia/reperfusion injury via suppression of PTEN and Biml expression," Oncotarget, vol. 7, no. 52, pp. 86926-86936, 2016.

[93] Z. Chen, S. Zhang, C. Guo, J. Li, and W. Sang, "Downregulation of miR-200c protects cardiomyocytes from hypoxia-induced apoptosis by targeting GATA-4," International Journal of Molecular Medicine, vol. 39, no. 6, pp. 1589-1596, 2017.

[94] B. Zhang, M. Zhou, C. Li et al., "MicroRNA-92a inhibition attenuates hypoxia/reoxygenation-induced myocardiocyte apoptosis by targeting Smad7," PLoS ONE, vol. 9, no. 6, Article ID e100298, 2014.

[95] C.-H. Yeh, T.-P. Chen, Y.-C. Wang, Y.-M. Lin, and S.-W. Fang, "MicroRNA-27a regulates cardiomyocytic apoptosis during cardioplegia-induced cardiac arrest by targeting interleukin 10related pathways," Shock, vol. 38, no. 6, pp. 607-614, 2012.

[96] H. Xiong, T. Luo, W. He et al., "Up-regulation of miR-138 inhibits hypoxia-induced cardiomyocyte apoptosis via downregulating lipocalin-2 expression," Experimental Biology and Medicine, vol. 241, no. 1, pp. 25-30, 2016.

[97] S. He, P. Liu, Z. Jian et al., "MiR-138 protects cardiomyocytes from hypoxia-induced apoptosis via MLK3/JNK/c-jun pathway," Biochemical and Biophysical Research Communications, vol. 441, no. 4, pp. 763-769, 2013.

[98] Y. Ye, Z. Hu, Y. Lin, C. Zhang, and J. R. Perez-Polo, "Downregulation of microRNA-29 by antisense inhibitors and a PPAR$\gamma$ agonist protects against myocardial ischaemia-reperfusion injury," Cardiovascular Research, vol. 87, no. 3, pp. 535-544, 2010.

[99] Z. W. Zhang, H. Li, S. S. Chen, Y. Li, Z. Y. Cui, and J. Ma, "MicroRNA-122 regulates caspase- 8 and promotes the apoptosis of mouse cardiomyocytes," Brazilian Journal of Medical and Biological Research, vol. 50, no. 2, p. e5760, 2017.

[100] I. Knezevic, A. Patel, N. R. Sundaresan et al., "A novel cardiomyocyte-enriched MicroRNA, miR-378, targets insulinlike growth factor 1 receptor: implications in postnatal cardiac remodeling and cell survival," The Journal of Biological Chemistry, vol. 287, no. 16, pp. 12913-12926, 2012.

[101] R.-Y. Zhu, D. Zhang, H.-D. Zou, X.-S. Zuo, Q.-S. Zhou, and H. Huang, "MiR-28 inhibits cardiomyocyte survival through suppressing PDK1/Akt/mTOR signaling," In Vitro Cellular and Developmental Biology-Animal, vol. 52, no. 10, pp. 1020-1025, 2016.

[102] Z. Pan, X. Sun, J. Ren et al., "miR-1 exacerbates cardiac ischemiareperfusion injury in mouse models," PLoS ONE, vol. 7, no. 11, Article ID e50515, 2012.

[103] H. Zhu, Y. Yang, Y. Wang, J. Li, P. W. Schiller, and T. Peng, "MicroRNA-195 promotes palmitate-induced apoptosis in cardiomyocytes by down-regulating Sirt1," Cardiovascular Research, vol. 92, no. 1, pp. 75-84, 2011.

[104] E. R. Porrello, B. A. Johnson, A. B. Aurora et al., "MiR-15 family regulates postnatal mitotic arrest of cardiomyocytes," Circulation Research, vol. 109, no. 6, pp. 670-679, 2011.

[105] S. P. Li, B. Liu, B. Song, C. X. Wang, and Y. C. Zhou, "Mir-28 promotes cardiac ischemia by targeting mitochondrial aldehyde dehydrogenase 2 (aldh2) in mus musculus cardiac myocytes," European Review for Medical and Pharmacological Sciences, vol. 19, pp. 752-758, 2015. 
[106] F. Fan, A. Sun, H. Zhao et al., "MicroRNA-34a promotes cardiomyocyte apoptosis post myocardial infarction through down-regulating aldehyde dehydrogenase 2," Current Pharmaceutical Design, vol. 19, no. 27, pp. 4865-4873, 2013.

[107] R. A. Boon, K. Iekushi, and S. Lechner, "MicroRNA-34a regulates cardiac ageing and function," Nature, vol. 494, no. 7439, pp. 107-110, 2013.

[108] H. Wu, F. Wang, S. Hu et al., "MiR-20a and miR-106b negatively regulate autophagy induced by leucine deprivation via suppression of ULK1 expression in C2C12 myoblasts," Cellular Signalling, vol. 24, no. 11, pp. 2179-2186, 2012.

[109] Z. Wang, N. Wang, P. Liu et al., "MicroRNA-25 regulates chemoresistance-associated autophagy in breast cancer cells, a process modulated by the natural autophagy inducer isoliquiritigenin," Oncotarget, vol. 5, no. 16, pp. 7013-7026, 2014.

[110] X. Duan, T. Zhang, S. Ding et al., "microRNA-17-5p modulates bacille calmette-guerin growth in RAW264.7 cells by targeting ULK1," PLoS ONE, vol. 10, no. 9, Article ID e0138011., 2015.

[111] Y. Huang, A. Y. Chuang, and E. A. Ratovitski, "Phospho$\Delta \mathrm{Np} 63 \alpha / \mathrm{miR}-885-3 \mathrm{p}$ axis in tumor cell life and cell death upon cisplatin exposure," Cell Cycle, vol. 10, no. 22, pp. 3938-3947, 2011.

[112] D. Z. John Clotaire, B. Zhang, N. Wei et al., "MiR-26b inhibits autophagy by targeting ULK2 in prostate cancer cells," Biochemical and Biophysical Research Communications, vol. 472, no. 1, pp. 194-200, 2016.

[113] B. Zheng, H. Zhu, D. Gu et al., "MiRNA-30a-mediated autophagy inhibition sensitizes renal cell carcinoma cells to sorafenib," Biochemical and Biophysical Research Communications, vol. 459, no. 2, pp. 234-239, 2015.

[114] R. Xu, S. Liu, H. Hong Chen, and L. Lao, "MicroRNA-30a downregulation contributes to chemoresistance of osteosarcoma cells through activating Beclin-1-mediated autophagy," Oncology Reports, vol. 35, no. 3, pp. 1757-1763, 2016.

[115] B. Wang, Y. Zhong, D. Huang, and J. Li, "Macrophage autophagy regulated by miR-384-5p-mediated control of Beclin-1 plays a role in the development of atherosclerosis," American Journal of Translational Research, vol. 8, no. 2, pp. 606-614, 2016.

[116] S. Tan, H. Shi, M. Ba et al., "MiR-409-3p sensitizes colon cancer cells to oxaliplatin by inhibiting Beclin-1-mediated autophagy," International Journal of Molecular Medicine, vol. 37, no. 4, pp. 1030-1038, 2016.

[117] X. Zhang, H. Shi, S. Lin, M. Ba, and S. Cui, "MicroRNA216a enhances the radiosensitivity of pancreatic cancer cells by inhibiting beclin-1-mediated autophagy," Oncology Reports, vol. 34, no. 3, pp. 1557-1564, 2015.

[118] Y. Huang, R. Guerrero-Preston, and E. A. Ratovitski, "Phospho$\Delta \mathrm{Np} 63 \alpha$-dependent regulation of autophagic signaling through transcription and micro-RNA modulation," Cell Cycle, vol. 11, no. 6, pp. 1247-1259, 2012.

[119] K. A. Tekirdag, G. Korkmaz, D. G. Ozturk, R. Agami, and D. Gozuacik, "MIR181A regulates starvation-and rapamycininduced autophagy through targeting of ATG5," Autophagy, vol. 9, no. 3, pp. 374-385, 2013.

[120] X. Guo, H. Xue, X. Guo et al., "MiR224-3p inhibits hypoxiainduced autophagy by targeting autophagy-related genes in human glioblastoma cells," Oncotarget, vol. 6, no. 39, pp. 4162041637, 2015.

[121] Y. Yu, L. Yang, M. Zhao et al., "Targeting microRNA-30amediated autophagy enhances imatinib activity against human chronic myeloid leukemia cells," Leukemia, vol. 26, no. 8, pp. 1752-1760, 2012.
[122] X. Yang, X. Zhong, J. L. Tanyi et al., "mir-30d regulates multiple genes in the autophagy pathway and impairs autophagy process in human cancer cells," Biochemical and Biophysical Research Communications, vol. 431, no. 3, pp. 617-622, 2013.

[123] Y. Chang, W. Yan, X. He et al., "MiR-375 inhibits autophagy and reduces viability of hepatocellular carcinoma cells under hypoxic conditions," Gastroenterology, vol. 143, no. 1, pp. 177$187,2012$.

[124] S. Zhao, D. Yao, J. Chen, N. Ding, and F. Ren, "MiR-20a promotes cervical cancer proliferation and metastasis in vitro and in vivo," PLoS ONE, vol. 10, no. 3, Article ID e0120905, 2015.

[125] Y. Zeng, G. Huo, Y. Mo, W. Wang, and H. Chen, "MIR137 Regulates Starvation-Induced Autophagy by Targeting ATG7," Journal of Molecular Neuroscience, vol. 56, no. 4, pp. 815-821, 2015.

[126] K. Wang, C.-Y. Liu, L.-Y. Zhou et al., "APF lncRNA regulates autophagy and myocardial infarction by targeting miR-188-3p," Nature Communications, vol. 6, article 6779, 2015.

[127] N. Xu, J. Zhang, C. Shen et al., "Cisplatin-induced downregulation of miR-199a-5p increases drug resistance by activating autophagy in HCC cell," Biochemical and Biophysical Research Communications, vol. 423, no. 4, pp. 826-831, 2012.

[128] G. Korkmaz, C. le Sage, K. A. Tekirdag, R. Agami, and D. Gozuacik, "miR-376b controls starvation and mTOR inhibition-related autophagy by targeting ATG4C and BECN1," Autophagy, vol. 8, no. 2, pp. 165-176, 2012.

[129] G. Korkmaz, K. A. Tekirdag, D. G. Ozturk, A. Kosar, O. U. Sezerman, and D. Gozuacik, "MIR376A is a regulator of starvation-induced autophagy," PLoS ONE, vol. 8, no. 12, Article ID e82556, 2013.

[130] L. B. Frankel, J. Wen, M. Lees et al., "microRNA-101 is a potent inhibitor of autophagy," The EMBO Journal, vol. 30, no. 22, pp. 4628-4641, 2011.

[131] G. Wang, T. Zhao, L. Wang et al., "Studying different binding and intracellular delivery efficiency of ssdna single-walled carbon nanotubes and their effects on lc3-related autophagy in renal mesangial cells via mirna-382," ACS Applied Materials and Interfaces, vol. 7, no. 46, pp. 25733-25740, 2015.

[132] Q. Jiang, Y. Han, H. Gao, R. Tian, P. Li, and C. Wang, "Ursolic acid induced anti-proliferation effects in rat primary vascular smooth muscle cells is associated with inhibition of microRNA-21 and subsequent PTEN/PI3K," European Journal of Pharmacology, vol. 781, pp. 69-75, 2016.

[133] V. Kovaleva, R. Mora, Y. J. Park et al., "miRNA-130a targets ATG2B and DICER1 to inhibit autophagy and trigger killing of chronic lymphocytic leukemia cells," Cancer Research, vol. 72, no. 7, pp. 1763-1772, 2012.

[134] Y. Jin, J. Wei, Z. Ma et al., "MiR-143 inhibits cell proliferation by targeting autophagy-related $2 \mathrm{~B}$ in non-small cell lung cancer H1299 cells," Molecular Medicine Reports, vol. 11, no. 1, pp. 571576, 2015.

[135] J. Huang, W. Sun, H. Huang et al., "MiR-34a modulates angiotensin II-induced myocardial hypertrophy by direct inhibition of ATG9A Expression and Autophagic Activity," PLoS ONE, vol. 9, no. 4, Article ID e94382, 2014.

[136] H. Zhai, B. Song, X. Xu, W. Zhu, and J. Ju, "Inhibition of autophagy and tumor growth in colon cancer by miR-502," Oncogene, vol. 32, no. 12, pp. 1570-1579, 2013.

[137] R. Wang, Z.-X. Wang, J.-S. Yang, X. Pan, W. De, and L.-B. Chen, "MicroRNA-451 functions as a tumor suppressor in human non-small cell lung cancer by targeting ras-related protein 14 (RAB14)," Oncogene, vol. 30, no. 23, pp. 2644-2658, 2011. 
[138] J. Tao, W. Liu, G. Shang et al., "MiR-207/352 regulate lysosomalassociated membrane proteins and enzymes following ischemic stroke," Neuroscience, vol. 305, pp. 1-14, 2015.

[139] A. Gombozhapova, Y. Rogovskaya, V. Shurupov et al., "Macrophage activation and polarization in post-infarction cardiac remodeling," Journal of Biomedical Science, vol. 24, no. 1, p. 13, 2017.

[140] M. Pennati, A. Lopergolo, and V. Profumo, "miR-205 impairs the autophagic flux and enhances cisplatin cytotoxicity in castration-resistant prostate cancer cells," Biochemical Pharmacology, vol. 87, no. 4, pp. 579-597, 2014.

[141] F. Xu, Y. H. Kang, H. Zhang et al., "Aktl comprised antibacterial response through regulating macrophage polarization," The Journal of Infectious Diseases, vol. 208, no. 3, pp. 528-538, 2013.

[142] Y. Zhang, M. Zhang, X. Li et al., "Silencing MicroRNA-155 attenuates cardiac injury and dysfunction in viral myocarditis via promotion of M2 phenotype polarization of macrophages," Scientific Reports, vol. 6, article 22613, 2016.

[143] A. C. Rothchild, J. R. Sissons, S. Shafiani et al., "MiR-155regulated molecular network orchestrates cell fate in the innate and adaptive immune response to Mycobacterium tuberculosis," Proceedings of the National Academy of Sciences of the United States of America, vol. 113, no. 41, pp. E6172-E6181, 2016.

[144] C. Wang, C. Zhang, L. Liu et al., "Macrophage-derived mir-155containing exosomes suppress fibroblast proliferation and promote fibroblast inflammation during cardiac injury," Molecular Therapy, vol. 25, no. 1, pp. 192-204, 2017.

[145] J. L. Bao and L. Lin, "Mir-155 and mir-148a reduce cardiac injury by inhibiting nf-kappab pathway during acute viral myocarditis," European Review for Medical and Pharmacological Sciences, vol. 18, pp. 2349-2356, 2014.

[146] X.-Q. Wu, Y. Dai, Y. Yang et al., "Emerging role of microRNAs in regulating macrophage activation and polarization in immune response and inflammation," Immunology, vol. 148, no. 3, pp. 237-248, 2016.

[147] S.-W. Kim, K. Ramasamy, H. Bouamar, A.-P. Lin, D. Jiang, and R. C. T. Aguiar, "MicroRNAs miR-125a and miR-125b constitutively activate the NF-kappaB pathway by targeting the tumor necrosis factor alpha-induced protein 3 (TNFAIP3, A20)," Proceedings of the National Academy of Sciences of the United States of America, vol. 109, no. 20, pp. 7865-7870, 2012.

[148] S. Banerjee, H. Cui, N. Xie et al., "miR-125a-5p regulates differential activation of macrophages and inflammation," The Journal of Biological Chemistry, vol. 288, no. 49, pp. 3542835436, 2013.

[149] C. I. Caescu, X. Guo, L. Tesfa et al., "Colony stimulating factor-1 receptor signaling networks inhibit mouse macrophage inflammatory responses by induction of microRNA-21," Blood, vol. 125, no. 8, pp. el-e13, 2015.

[150] W. Ying, A. Tseng, R. C.-A. Chang et al., "MicroRNA-223 is a crucial mediator of PPAR $\gamma$-regulated alternative macrophage activation," Journal of Clinical Investigation, vol. 125, no. 11, pp. 4149-4159, 2015.

[151] F. Yao, Y. Yu, L. Feng et al., "Adipogenic miR-27a in adipose tissue upregulates macrophage activation via inhibiting PPAR $\gamma$ of insulin resistance induced by high-fat diet-associated obesity," Experimental Cell Research, vol. 355, pp. 105-112, 2017.

[152] V. N. Garikipati, S. K. Verma, D. Jolardarashi et al., “Therapeutic inhibition of miR-375 attenuates post-myocardial infarction inflammatory response and left ventricular dysfunction via PDK-1-AKT signalling axis," Cardiovascular Research, vol. 113, no. 8, pp. 938-949, 2017.
[153] S. Suresh Babu, R. A. Thandavarayan, D. Joladarashi et al., "MicroRNA-126 overexpression rescues diabetes-induced impairment in efferocytosis of apoptotic cardiomyocytes," Scientific Reports, vol. 6, Article ID 36207, 2016.

[154] M. Yuan, L. Zhang, F. You et al., "MiR-145-5p regulates hypoxiainduced inflammatory response and apoptosis in cardiomyocytes by targeting CD40," Molecular and Cellular Biochemistry, vol. 431, pp. 123-131, 2017.

[155] M. Gao, X. Wang, X. Zhang et al., "Attenuation of cardiac dysfunction in polymicrobial sepsis by MicroRNA-146a is mediated via targeting of IRAK1 and TRAF6 expression," Journal of Immunology, vol. 195, no. 2, pp. 672-682, 2015.

[156] D. A. Chistiakov, A. N. Orekhov, and Y. V. Bobryshev, "The role of miR-126 in embryonic angiogenesis, adult vascular homeostasis, and vascular repair and its alterations in atherosclerotic disease," Journal of Molecular and Cellular Cardiology, vol. 97, pp. 47-55, 2016.

[157] C.-Y. Chen, C.-M. Su, C.-J. Hsu et al., "CCN1 promotes vegf production in osteoblasts and induces endothelial progenitor cell angiogenesis by inhibiting mir-126 expression in rheumatoid arthritis," Journal of Bone and Mineral Research, vol. 32, no. 1, pp. 34-45, 2017.

[158] Y. Chen and D. H. Gorski, "Regulation of angiogenesis through a microRNA (miR-130a) that down-regulates antiangiogenic homeobox genes GAX and HOXA5," Blood, vol. 111, no. 3, pp. 1217-1226, 2008.

[159] C. Lu, X. Wang, T. Ha et al., "Attenuation of cardiac dysfunction and remodeling of myocardial infarction by microRNA130a are mediated by suppression of PTEN and activation of PI3K dependent signaling," Journal of Molecular and Cellular Cardiology, vol. 89, pp. 87-97, 2015.

[160] J. T. Mendell, "miRiad roles for the miR-17-92 cluster in development and disease," Cell, vol. 133, no. 2, pp. 217-222, 2008.

[161] P. Fasanaro, Y. D’Alessandra, V. Di Stefano et al., "MicroRNA210 modulates endothelial cell response to hypoxia and inhibits the receptor tyrosine kinase ligand ephrin-A3," Journal of Biological Chemistry, vol. 283, no. 23, pp. 15878-15883, 2008.

[162] K. Pulkkinen, T. Malm, M. Turunen, J. Koistinaho, and S. YläHerttuala, "Hypoxia induces microRNA miR-210 in vitro and in vivo," FEBS Letters, vol. 582, no. 16, pp. 2397-2401, 2008.

[163] T. Würdinger, B. A. Tannous, O. Saydam et al., "miR-296 regulates growth factor receptor overexpression in angiogenic endothelial cells," Cancer Cell, vol. 14, no. 5, pp. 382-393, 2008.

[164] D. Y. Lee, Z. Deng, C. H. Wang, and B. B. Yang, "MicroRNA378 promotes cell survival, tumor growth, and angiogenesis by targeting SuFu and Fus-1 expression," Proceedings of the National Academy of Sciences of the United States of America, vol. 104, no. 51, pp. 20350-20355, 2007.

[165] C. Templin, J. Volkmann, M. Y. Emmert et al., "Increased proangiogenic activity of mobilized CD34 + progenitor cells of patients with acute ST-segment-elevation myocardial infarction: Role of differential MicroRNA-378 expression," Arteriosclerosis, Thrombosis, and Vascular Biology, vol. 37, no. 2, pp. 341-349, 2017.

[166] S. Anand, B. K. Majeti, L. M. Acevedo et al., "MicroRNA-132mediated loss of p120RasGAP activates the endothelium to facilitate pathological angiogenesis," Nature Medicine, vol. 16, no. 8, pp. 909-914, 2010.

[167] Q. Zhou, R. Gallagher, R. Ufret-Vincenty, X. Li, E. N. Olson, and S. Wang, "Regulation of angiogenesis and choroidal neovascularization by members of microRNA-23 27 24 clusters," 
Proceedings of the National Academy of Sciences of the United States of America, vol. 108, no. 20, pp. 8287-8292, 2011.

[168] D. Veliceasa, D. Biyashev, G. Qin et al., “Therapeutic manipulation of angiogenesis with miR-27b," Vascular Cell, vol. 7, no. 1, article 6, 2015.

[169] J. Fiedler, V. Jazbutyte, B. C. Kirchmaier et al., "MicroRNA-24 regulates vascularity after myocardial infarction," Circulation, vol. 124, no. 6, pp. 720-730, 2011.

[170] M. Meloni, M. Marchetti, K. Garner et al., "Local inhibition of microRNA-24 improves reparative angiogenesis and left ventricle remodeling and function in mice with myocardial infarction," Molecular Therapy, vol. 21, no. 7, pp. 1390-1402, 2013.

[171] Q. Duan, L. Yang, W. Gong et al., "MicroRNA-214 is upregulated in heart failure patients and suppresses XBP1-mediated endothelial cells angiogenesis," Journal of Cellular Physiology, vol. 230, no. 8, pp. 1964-1973, 2015.

[172] B. C. Bernardo, X.-M. Gao, C. E. Winbanks et al., “Therapeutic inhibition of the miR-34 family attenuates pathological cardiac remodeling and improves heart function," Proceedings of the National Academy of Sciences of the United States of America, vol. 109, no. 43, pp. 17615-17620, 2012.

[173] T. Ito, S. Yagi, and M. Yamakuchi, "MicroRNA-34a regulation of endothelial senescence," Biochemical and Biophysical Research Communications, vol. 398, no. 4, pp. 735-740, 2010.

[174] T. Zhao, J. Li, and A. F. Chen, "MicroRNA-34a induces endothelial progenitor cell senescence and impedes its angiogenesis via suppressing silent information regulator 1," American Journal of Physiology-Endocrinology and Metabolism, vol. 299, no. 1, pp. E110-E116, 2010.

[175] L. Xiao, H. He, L. Ma et al., "Effects of mir-29a and mir101a expression on myocardial interstitial collagen generation after aerobic exercise in myocardial-infarcted rats," Archives of Medical Research, vol. 48, no. 1, pp. 27-34, 2017.

[176] L. P. Zhu, J. P. Zhou, J. X. Zhang et al., "Mir-15b-5p regulates collateral artery formation by targeting akt3 (protein kinase b3)," Arteriosclerosis, Thrombosis, and Vascular Biology, vol. 37, no. 5, pp. 957-968, 2017.

[177] S. Grundmann, F. P. Hans, S. Kinniry et al., "MicroRNA100 regulates neovascularization by suppression of mammalian target of rapamycin in endothelial and vascular smooth muscle cells," Circulation, vol. 123, no. 9, pp. 999-1009, 2011.

[178] C. P. Bracken, P. A. Gregory, and N. Kolesnikoff, "A doublenegative feedback loop between ZEB1-SIP1 and the microRNA200 family regulates epithelial-mesenchymal transition," Cancer Research, vol. 68, no. 19, pp. 7846-7854, 2008.

[179] P. A. Gregory, A. G. Bert, E. L. Paterson et al., "The miR200 family and miR-205 regulate epithelial to mesenchymal transition by targeting ZEB1 and SIP1," Nature Cell Biology, vol. 10, no. 5, pp. 593-601, 2008.

[180] Y. C. Chan, S. Roy, S. Khanna, and C. K. Sen, "Downregulation of endothelial microRNA-200b supports cutaneous wound angiogenesis by desilencing GATA binding protein 2 and vascular endothelial growth factor receptor 2," Arteriosclerosis, Thrombosis, \& Vascular Biology, vol. 32, no. 6, pp. 1372-1382, 2012.

[181] Y.-C. Choi, S. Yoon, Y. Jeong, J. Yoon, and K. Baek, "Regulation of vascular endothelial growth factor signaling by miR-200b," Molecules and Cells, vol. 32, no. 1, pp. 77-82, 2011.

[182] A. Caporali, M. Meloni, C. Völlenkle et al., "Deregulation of microRNA-503 contributes to diabetes mellitus-induced impairment of endothelial function and reparative angiogenesis after limb ischemia," Circulation, vol. 123, no. 3, pp. 282-291, 2011.

[183] K.-J. Yin, K. Olsen, M. Hamblin, J. Zhang, S. P. Schwendeman, and Y. E. Chen, "Vascular endothelial cell-specific MicroRNA15a inhibits angiogenesis in hindlimb ischemia," Journal of Biological Chemistry, vol. 287, no. 32, pp. 27055-27064, 2012.

[184] A. Chamorro-Jorganes, E. Araldi, L. O. F. Penalva, D. Sandhu, C. Fernández-Hernando, and Y. Suárez, "MicroRNA-16 and MicroRNA-424 regulate cell-autonomous angiogenic functions in endothelial cells via targeting vascular endothelial growth factor receptor-2 and fibroblast growth factor receptor-1," Arteriosclerosis, Thrombosis, and Vascular Biology, vol. 31, no. 11, pp. 2595-2606, 2011.

[185] X. H. Wang, R. Z. Qian, W. Zhang, S. F. Chen, H. M. Jin, and R. M. Hu, "MicroRNA-320 expression in myocardial microvascular endothelial cells and its relationship with insulin-like growth factor-1 in type 2 diabetic rats," Clinical and Experimental Pharmacology \& Physiology, vol. 36, no. 2, pp. 181-188, 2009.

[186] X. Wang, W. Huang, G. Liu et al., "Cardiomyocytes mediate anti-angiogenesis in type 2 diabetic rats through the exosomal transfer of miR-320 into endothelial cells," Journal of Molecular and Cellular Cardiology, vol. 74, pp. 139-150, 2014.

[187] P. Wang, Y. Luo, H. Duan et al., "MicroRNA 329 Suppresses Angiogenesis by Targeting CD146," Molecular and Cellular Biology, vol. 33, no. 18, pp. 3689-3699, 2013.

[188] C. Ohyagi-Hara, K. Sawada, S. Kamiura et al., "MiR-92a inhibits peritoneal dissemination of ovarian cancer cells by inhibiting integrin $\alpha 5$ expression," The American Journal of Pathology, vol. 182, no. 5, pp. 1876-1889, 2013.

[189] A. Bonauer, G. Carmona, M. Iwasaki et al., "MicroRNA-92a controls angiogenesis and functional recovery of ischemic tissues in Mice," Science, vol. 324, no. 5935, pp. 1710-1713, 2009.

[190] R. Hinkel, D. Penzkofer, S. Zühlke et al., "Inhibition of microRNA-92a protects against ischemia/reperfusion injury in a large-animal model," Circulation, vol. 128, no. 10, pp. 10661075, 2013.

[191] Z. Jing, W. Han, X. Sui, J. Xie, and H. Pan, "Interaction of autophagy with microRNAs and their potential therapeutic implications in human cancers," Cancer Letters, vol. 356, no. 2, pp. 332-338, 2015.

[192] L. Bao, L. Lv, J. Feng et al., "Mir-487b-5p regulates temozolomide resistance of lung cancer cells through lamp2-medicated autophagy," DNA and Cell Biology, vol. 35, no. 8, pp. 385-392, 2016.

[193] Y. Lim and S. Kumar, "A single cut to pyroptosis," Oncotarget, vol. 6, no. 35, pp. 36926-36927, 2015.

[194] P. Jeyabal, R. A. Thandavarayan, D. Joladarashi et al., "MicroRNA-9 inhibits hyperglycemia-induced pyroptosis in human ventricular cardiomyocytes by targeting ELAVL1," Biochemical and Biophysical Research Communications, vol. 471, no. 4, pp. 423-429, 2016.

[195] F. Bauernfeind, A. Rieger, F. A. Schildberg, P. A. Knolle, J. L. Schmid-Burgk, and V. Hornung, "NLRP3 inflammasome activity is negatively controlled by miR-223," Journal of Immunology, vol. 189, no. 8, pp. 4175-4181, 2012.

[196] P. Carmeliet, "Angiogenesis in life, disease and medicine," Nature, vol. 438, no. 7070, pp. 932-936, 2005.

[197] J. S. Esser, E. Saretzki, F. Pankratz et al., "Bone morphogenetic protein 4 regulates microRNAs miR-494 and miR-126-5p in control of endothelial cell function in angiogenesis," Thrombosis and Haemostasis, vol. 117, no. 4, pp. 734-749, 2017. 
[198] C. Doebele, A. Bonauer, A. Fischer et al., "Members of the microRNA-17-92 cluster exhibit a cell-intrinsic antiangiogenic function in endothelial cells," Blood, vol. 115, no. 23, pp. 49444950, 2010.

[199] M. Dews, A. Homayouni, D. Yu et al., "Augmentation of tumor angiogenesis by a Myc-activated microRNA cluster," Nature Genetics, vol. 38, no. 9, pp. 1060-1065, 2006. 


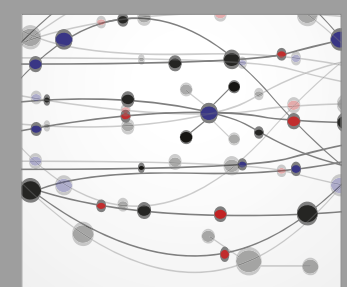

The Scientific World Journal
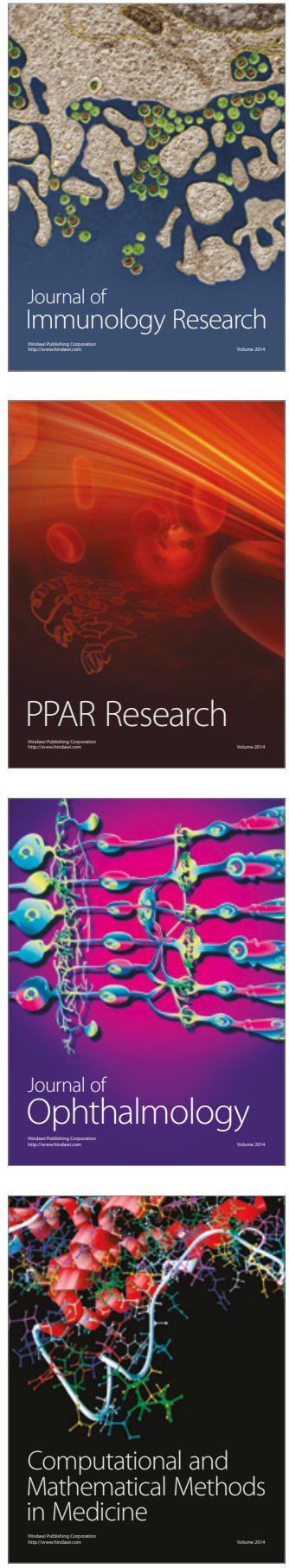

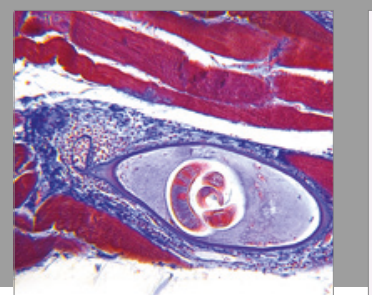

Gastroenterology Research and Practice
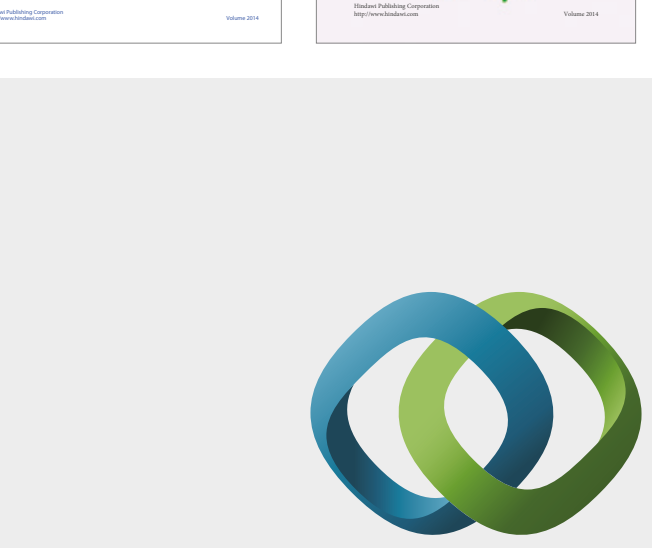

\section{Hindawi}

Submit your manuscripts at

https://www.hindawi.com
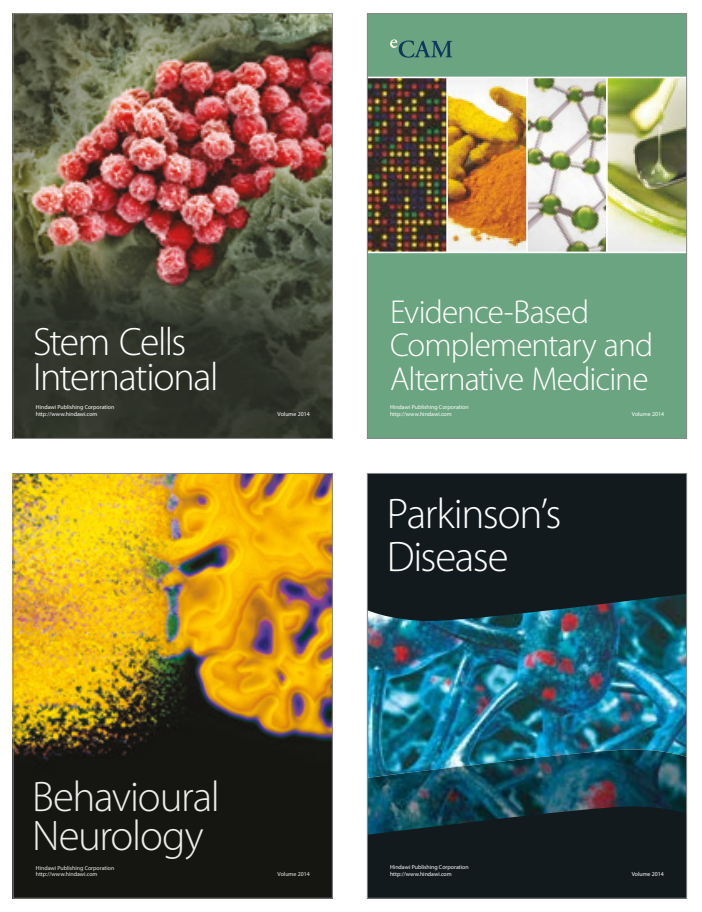
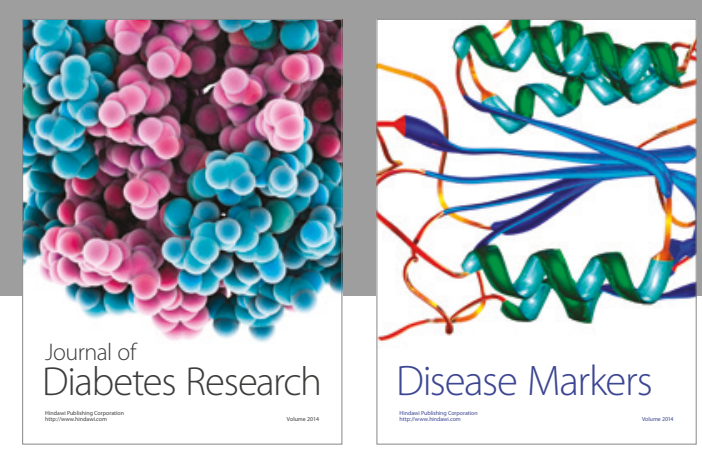

Disease Markers
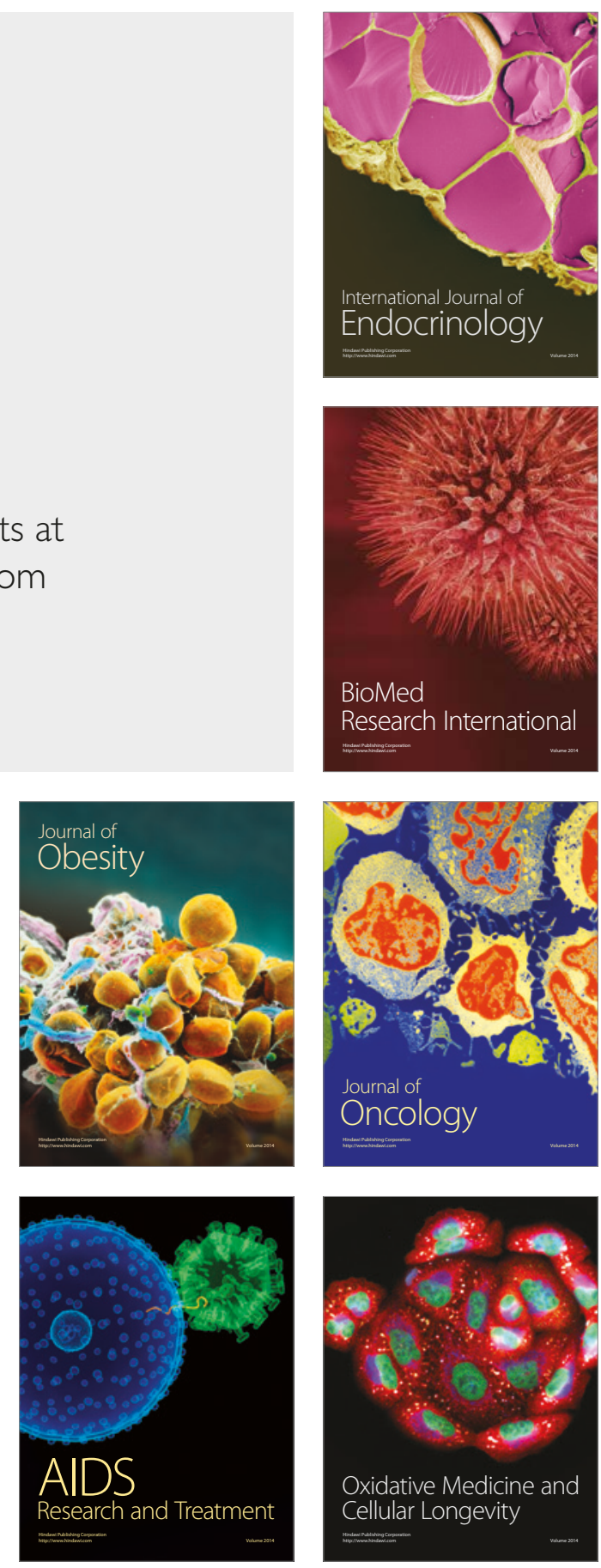